\author{
UNIVERSIDADE DE SÃO PAULO \\ FACULDADE DE CIÊNCIAS FARMACÊUTICAS DE RIBEIRÃO PRETO
}

\title{
Estudo da utilização de medicamentos em usuários portadores de diabetes mellitus atendidos pelo sistema único de saúde
}

Camilo Molino Guidoni 


\section{UNIVERSIDADE DE SÃO PAULO \\ FACULDADE DE CIÊNCIAS FARMACÊUTICAS DE RIBEIRÃO PRETO}

\section{Estudo da utilização de medicamentos em usuários portadores de diabetes mellitus atendidos pelo sistema único de saúde}

Dissertação de Mestrado apresentada ao Programa de Pós-Graduação em Ciências Farmacêuticas para obtenção do Título de Mestre em Ciências Farmacêuticas.

Área de concentração: Medicamentos e Cosméticos.

Orientado: Camilo Molino Guidoni Orientador: Prof. Dr. Leonardo R. L. Pereira

Ribeirão Preto-SP 




\section{RESUMO}

GUIDONI, C.M; PEREIRA, L.R.L. Estudo da utilização de medicamentos em usuários portadores de diabetes mellitus atendidos pelo sistema único de saúde. 2009. 160f. Dissertação (Mestrado). Faculdade de Ciências Farmacêuticas de Ribeirão Preto, Universidade de São Paulo, Ribeirão Preto, 2009.

O Diabetes mellitus é uma das doenças crônicas não-transmissíveis mais prevalentes do mundo, com prevalência estimada para 2025 de $7,1 \%$ e $14,0 \%$ da população mundial e brasileira, respectivamente. Atualmente existe a necessidade de desenvolvimento de programas com ênfase na prevenção primária, controle da incidência e complicações do diabetes devido ao elevado índice de morbimortalidade, associação com comorbidades e custo social-econômico. Para isso, uma das possíveis estratégias seria avaliar a utilização de medicamentos antidiabéticos orais e insulina prescritos aos portadores de DM atendidos pelo Sistema Único de Saúde. No presente trabalho, os usuários diabéticos pertencentes à rede municipal de saúde do Distrito Sanitário Oeste de Ribeirão Preto-SP foram identificados pela retirada de medicamentos antidiabéticos orais ou insulina nas farmácias das Unidades de Saúde do Distrito através da base de dados da prefeitura, denominado sistema Hygia de informação. Dessa forma, foi possível avaliar a terapia farmacológica para o tratamento do diabetes, a freqüência de retirada dos antidiabéticos orais e insulina, análise da outras classes medicamentosas prescritas para o controle das outras enfermidades e acesso aos diversos níveis de atenção à saúde. Por meio do banco de dados Hygia foram identificados 3927 usuários portadores de diabetes pertencentes ao Distrito Oeste de Ribeirão Preto-SP no período de mar/2006 a fev/2007, sendo a média de idade de 60,4 anos e $60,8 \%$ dos usuários identificados pertencerem ao gênero feminino. A monoterapia $(60,0 \%)$ foi o esquema terapêutico mais prescrito, sendo a metformina o medicamento mais utilizado (31,8\%). Apenas 3,3\% dos usuários utilizaram insulina de forma isolada. No geral, as doses dos medicamentos antidiabéticos orais foram mais elevadas em politerapia do que em monoterapia, sendo que a freqüência média de retirada foi superior a $70,0 \%$. Aproximadamente $34,0 \%$ dos indivíduos apresentaram insucesso no tratamento farmacológico do diabetes, com adição de medicamentos e/ou aumento da dose. Medicamentos como hidroclorotiazida, cimetidina, diclofenaco, entre outros, devem ser administrados com cautela em pacientes portadores de diabetes devido à possibilidade de elevar o risco de alteração da glicemia. Em relação às comorbidades, $73,5 \%$ dos usuários identificados são hipertensos e $25,9 \%$ dislipidêmicos. O número médio de consultas durante o período de estudo foi de 7,0 para todos os Grupos Terapêuticos. Portanto, através do estudo do perfil epidemiológico do Diabetes mellitus, foi possível verificar que existe a necessidade de se conhecer a situação real do consumo de medicamentos e do perfil epidemiológico das populações, além de constatar que os estudos de utilização de medicamentos são uma ferramenta útil neste processo, contribuindo para o uso racional dos medicamentos, direcionamento da prática médica e formulação de políticas de saúde.

Palavras-chave: Sistema Único de Saúde, Farmacoepidemiologia, Estudos de Utilização de Medicamentos, Diabetes mellitus, Doenças Crônicas NãoTransmissíveis. 


\begin{abstract}
GUIDONI, C.M; PEREIRA, L.R.L. Drug utilization study in diabetic patients attends by public health system. 2009. 160f Dissertation (Master). Faculdade de Ciências Farmacêuticas de Ribeirão Preto, Universidade de São Paulo, Ribeirão Preto, 2009.
\end{abstract}

The Diabetes mellitus is one of the non-transmissible chronic diseases most prevalent in the world. It is estimated that diabetes affect more than $7.1 \%$ people worldwide and $14.0 \%$ people in Brazil by 2025 . Currently, it is necessary to develop programs with emphasis in the primary prevention, incidence and complications diabetes control due to the high mortality and comorbities rate. Therefore, one of the possible strategies would be to evaluate the use of oral antidiabetic agents and insulin prescribed to the people with DM attends by brazilian public health system. In the present study, the diabetic patients belonging to the public health system of the West Sanitary District of Ribeirão Preto-SP were identified to receive orals agents or insulin from pharmacy of the units of public health system of the West District through the Hygia database record. So, it was possible to evaluate the pharmacological therapy for the treatment of the diabetes, the frequency of oral agents and insulin received, analysis of the other medicines classes prescribed to control comorbities and access at the public health system. In this study was identified 3927 patient with diabetes attend to the West District of Ribeirão Preto-SP in the period from March 2006 to February 2007. The mean age of the patient population was 60.4 years and $60.8 \%$ of patients were females. The most frequent treatment prescribed was monotherapy $(60.0 \%)$ and the metformin was drug more prescribed $(31.8 \%)$. Only $3.3 \%$ of the patients were treated with insulin in monotherapy. In general, the doses of the oral antidiabetic agents were higher in politherapy than monotherapy, and the mean frequency of drugs received was higher to $70.0 \%$. Approximately $34.0 \%$ of the patients showed failure in the pharmacological treatment of the diabetes, with addition of drugs and/or dose increase. Medicines such as hydrochlorothiazide, cimetidine, diclofenac, and others, should be administered with caution in patient with diabetes due to the possibility of increasing the risk of glycemic alteration. In relation to comorbities, $73.5 \%$ of the identified patients are hypertensive and $25.9 \%$ dyslipidemic. The mean of consultations was 7.0 in all Therapeutic Groups. Therefore, through of the study of the epidemic Diabetes mellitus profile, it was possible to verify that it is necessary to know the real situation of the consumption of medicines and the epidemic population profile, besides to verify that the drug utilization studies are a useful tool in this process, contributing to the rational use of the medicines, direction of the medical practice and politics health formulation.

Keywords: Public Health System, Pharmacoepidemioloy, Drug Utilization Study, Diabetes mellitus, Non-Transmissible Chronic Disease. 


\section{INTRODUÇÃO}

"O desejo de tomar o medicamento é, talvez, a principal característica que distingue o homem dos outros animais" (William Osler). 


\subsection{O SISTEMA ÚNICO DE SAÚDE}

A criação do Sistema Único de Saúde (SUS) foi um marco histórico na sociedade brasileira, pois, pela primeira vez a sociedade civil participou da formulação do sistema de saúde do Brasil. Na verdade, a criação do SUS transcende a área da saúde e passa a representar toda uma movimentação social e inquietudes vividas pela sociedade nas décadas de 1970 e 1980, durante o regime militar, autoritarista, classicista e repressor.

A concepção desse sistema de saúde acontece em meio à crise políticoideológica e fiscal da ditadura militar onde se inicia processos de mudanças no regime governamental, culminando na transformação da política brasileira em democracia representativa formal (MS, 2006b).

Essas mudanças podem ser exemplificadas, segundo Rodriguez Neto (1988), com a realização do I Simpósio sobre Política Nacional de Saúde, ocorrido na câmara dos deputados em 1979, onde pela primeira vez se apresentou, de forma "pública, extensa e bastante consensual", a proposta do SUS, com um pensamento crítico oposicionista-reformista apontando a crise do sistema de saúde vigente do país, bem como a necessidade de democratização da sociedade, como requisito de sua superação.

Além disso, no início da década de 1980, em meio ao cenário de contradições e crise, emerge na sociedade brasileira o Movimento da Reforma Sanitarista ou Movimento Sanitarista definido como

conjunto organizado de pessoas e grupos partidários ou não articulados ao redor de um projeto (ESCOREL, 1998), cujo desenho e conteúdo foram sendo construídos ao longo do tempo a partir de um conjunto de práticas que Arouca (1976) caracterizou em três níveis: a prática teórica (construção do saber), a prática ideológica (transformação da consciência) e a prática política (transformação das relações sociais) (CARVALHO, 1995).

Este movimento, vindo de algumas experiências da década anterior, propõe como linha tática a ocupação dos espaços institucionais e a formulação ativa de políticas de saúde frente à crise previdenciária do Estado. Foi conferido ao movimento sanitário o papel de agente "portador coletivo e obstinado da participação institucionalizada e permanente da sociedade na gestão do sistema de saúde, como elemento estratégico do processo de reforma da saúde" (CARVALHO, 1995). 
A convocação da VIII Conferência Nacional de Saúde, em 1986, é considerada fato histórico na reforma sanitária brasileira, pois nela foi recomendada a reestruturação do Sistema Nacional de Saúde e, diferentemente das sete anteriores, apresentou grande representatividade social. As propostas traçadas constituíram arcabouço jurídico do sistema de saúde brasileiro constitucionalizadas em 1988, com a conquista do direito à saúde com controle social (MS, 2006b), resultando na criação do SUS. Este deveria ter comando único em cada esfera de governo, reforçando o poder político, administrativo e financeiro dos Estados e Municípios (MARIN et al., 2003).

Essa reforma, dada num processo complexo de lutas, negociações, arranjos políticos, teve sua base jurídico-institucional na Constituição Federal de 1988 e nas leis subseqüentes, onde foram definidos os princípios éticos/doutrinários do SUS, sendo eles: integralidade, universalidade e eqüidade. A competência para cuidar da saúde deixa de ser hegemônica da União, iniciando grandes mudanças estruturais, particularmente no processo de descentralização (BRASIL, 1988). As leis 8080/90 e 8142/90 consagram os princípios da descentralização dos serviços de saúde e municipalização da gestão, definindo papéis e atribuições dos gestores nas três esferas de atuação. Pode-se destacar que 0 artigo $6^{\circ}$ da Lei $n^{\circ} 8080$ "assegura 0 provimento da Assistência Terapêutica integral, incluindo a Assistência Farmacêutica" (BRASIL, 1990a; BRASIL, 1990b; Grifo nosso).

Em 2006, o SUS possuía em torno de 63 mil unidades ambulatoriais, 6 mil unidades hospitalares, com mais de 440 mil leitos, respondendo por aproximadamente 1 bilhão de procedimentos de atenção primária à saúde, destacando-se as consultas médicas (150 milhões) e os exames laboratoriais (300 milhões), assim como atendimentos de alta complexidade (132 milhões), internações hospitalares (12 milhões), partos (2 milhões) e 14 mil transplantes de órgãos (BRASIL, 2006a). Entretanto, a política de controle e prevenção das doenças crônicas não-transmissíveis (DCNT) ainda pode ser considerada incipiente.

O SUS foi o primeiro modelo brasileiro de sistema de saúde a adotar a Assistência Farmacêutica (AF) e a Política Nacional de Medicamentos (PNM) como instrumentos estratégicos na formulação das políticas de saúde, possibilitando ao farmacêutico não só participar de maneira mais efetiva da saúde pública, mas também desenvolver formas específicas de tecnologias envolvendo os medicamentos e a prestação de serviços de saúde (MARIN et al., 2003). Em 2002, a 
Portaria no 371/GM criou o Programa Nacional de Assistência Farmacêutica para Diabetes Mellitus (DM), como parte integrante do Plano Nacional de Reorganização da Atenção à Hipertensão Arterial Sistêmica (HAS) e DM, garantindo aos usuários cadastrados nos municípios os seguintes medicamentos: glibenclamida 5,0 mg, metformina 850,0 mg e insulina humana regular e NPH 100UI. (BRASIL, 2002; MS, 2002a; MS, 2007a). A Secretaria Municipal de Saúde de Ribeirão Preto-SP (SMSRP) disponibiliza para o tratamento dos portadores de DM, além dos três medicamentos listados acima, o medicamento glicazida $80,0 \mathrm{mg}$.

Os medicamentos constituem a tecnologia mais utilizada no tratamento das DCNT, sendo o item mais oneroso para os sistemas de saúde. Além disso, os portadores de DCNT necessitam de tratamento farmacológico por toda a vida e atualmente existe uma preocupação crescente dos sistemas de saúde do mundo e do Brasil com a elevação dos custos com insumos farmacêuticos.

No Canadá, o investimento na aquisição de medicamentos aumentou 11,0\% em 2005, alcançando US\$ 25 bilhões, sendo considerada a segunda maior despesa em saúde, superada apenas pelos gastos hospitalares (KONDRO, 2006). No Reino Unido, o custo com medicamentos cresceu 12,0\% em 2002, sendo que 25,0\% deste aumento foi devido à aquisição de apenas quatro classes terapêuticas: antidislipidêmicos, antidiabéticos, anti-hipertensivos e antipsicóticos (MACDONALD, 2003).

No Brasil, revela-se preocupante a evolução do custo dos medicamentos devido ao aumento percentual do orçamento anual do Ministério da Saúde (MS). Enquanto os gastos totais com saúde aumentaram em 9,6\%, aqueles com insumos farmacêuticos tiveram incremento de 123,9\% no período de 2002 a 2006, evidenciando o descompasso do crescimento (MS, 2007b).

Tal fato demonstra que a utilização dos medicamentos precisa ser monitorada para identificar fatores que possam minimizar essa discrepância. Em 2006, segundo o Instituto Brasileiro de Geografia e Estatística (IBGE), o Produto Interno Bruto (PIB) brasileiro cresceu 3,7\% enquanto houve aumento real de 7,5\% no gasto do MS e de 26,0\% para aquisição de medicamentos, sendo assim a União teve que ampliar o repasse financeiro para o MS que, por sua vez, possivelmente teve que reduzir investimentos em outros setores (MS, 2007b).

Devido a essa realidade, os estudos de utilização de medicamentos (EUMs) tornam-se fundamentais para detectar situações de risco para a população e 
monitorar o consumo dos medicamentos. Além disso, essa categoria de estudo demonstra eficiência mundialmente confirmada através de resultados obtidos anteriormente, tais como a retirada do mercado de vários medicamentos, sendo o mais atual a proibição da comercialização dos antiinflamatórios não-esteroidais com bloqueio seletivo para enzima ciclooxigenase 2 (PSATY, FURBERG, 2005).

Contudo, mesmo os EUMs sendo considerados menos dispendiosos financeiramente que outros tipos de estudos e importantes na análise do uso dos medicamentos na população "real", ainda continuam sendo escassos em nosso país.

\subsection{A FARMACOEPIDEMIOLOGIA}

No final do século XV já existia a preocupação com o uso dos medicamentos na sociedade, sendo conhecido que a utilização inadequada de qualquer substância química poderia resultar em efeitos contrários aos pretendidos, mesmo em doses "terapêuticas". Exemplificando, Paracelsus (1493-1541) descreve que "todas as substâncias são venenos, não há uma que não seja veneno. A dose diferencia o veneno do remédio" (KLAASSEN, 2006).

No início do século XX, a maioria dos medicamentos disponíveis para a sociedade era de origem natural e muitas vezes de natureza e estrutura química desconhecidas (LAPORTE, TOGNONI, ROSENFELD, 1989). Neste momento, a comprovação da eficiência terapêutica era a maior preocupação das autoridades sanitárias para permitir o consumo dos medicamentos pela população.

Entretanto, a partir da década de 1930, após um acidente que produziu mais de 100 mortes devido ao uso de dietilenoglicol como excipiente de um xarope de sulfanilamida nos Estados Unidos, as autoridades sanitárias norte-americanas começaram a se preocupar com os problemas das reações adversas aos medicamentos (RAM) (ROZENFELD, RANGEL, 1988), percebendo a necessidade de revisão das normas de permissão de comercialização desses insumos.

Com a "explosão farmacológica" ocorrida em meados da década de 1940, principalmente após a II Guerra Mundial, ocorre uma alteração no consumo dos medicamentos, principalmente devido ao desenvolvimento de novos fármacos sintéticos que possibilitou o aumento do arsenal terapêutico disponível, no entanto, 
houve também uma ascensão da ocorrência de acidentes de diferentes gravidades relacionadas à utilização dos medicamentos.

O episódio sobre RAM mais conhecida do mundo ocorreu com a talidomida, em 1961, responsável por um surto de malformação congênita rara, denominada focomelia, que acometeu aproximadamente 4 mil recém nascidos, ocasionando 498 mortes (LENZ, 1989). A partir desse fato, vários países do mundo iniciaram ações e atitudes com a finalidade de aperfeiçoar o sistema de registro de novos medicamentos, bem como da utilização destes, exigindo estudos mais completos das indústrias farmacêuticas.

O fato histórico da talidomida evidenciou claramente a necessidade de se complementar as informações da pesquisa de farmacologia básica, exigidas para lançamento do produto no mercado, com as informações provenientes da farmacologia clínica, que envolve o uso do medicamento em condições reais, principalmente em grupos de pessoas que são excluídos (idosos, crianças e gestantes) dos ensaios clínicos tradicionais, realizados nos períodos de précomercialização.

A partir desse momento, começaram a ser exigidos da indústria farmacêutica estudos de Farmacovigilância, denominados estudos de Fase IV. Sendo assim, a Farmacovigilância foi definida como:

- “identificação e avaliação dos efeitos, agudos ou crônicos, do risco do uso dos tratamentos farmacológicos no conjunto da população ou em grupos de pacientes expostos a tratamentos específicos" (LAPORTE, TOGNONI, ROSENFELD, 1989).

- "uma ciência relativa à detecção, avaliação, compreensão e prevenção dos efeitos adversos ou quaisquer problemas relacionados a medicamentos" (OMS, 2002).

A Farmacovigilância foi criada tendo como principal objetivo o estudo dos possíveis riscos advindos da utilização dos medicamentos pela população. Aliado a essa nova área do conhecimento surge, inserida no contexto da Farmacologia Clínica e da Epidemiologia, uma nova corrente de estudo, denominada Farmacoepidemiologia, que além dos estudos relacionados à segurança do medicamento, também possui como objetivo principal a descrição e o controle da utilização dos medicamentos em populações definidas num certo tempo e espaço (STROM, 2000).

Deste modo, a Farmacoepidemiologia é considerada um campo de estudo que constitui uma ponte entre a farmacologia, a terapêutica, a epidemiologia e a 
estatística (HARTZEMA, PORTA, HUGH, 1998). Sendo assim, a Farmacovigilância passa a ser uns dos objetos de estudo da Farmacoepidemiologia, que descreve a utilização e os padrões de prescrição dos medicamentos na população humana na pós-comercialização.

A conceituação do termo Farmacoepidemiologia evoluiu ao passar dos anos, sendo descrito por vários autores conforme demonstrado abaixo:

- Segundo Spitzer (WERTHEIMER, ANDREW, 1995)

é o estudo dos medicamentos como determinantes da saúde e da doença na população em geral. Isso inclui medir os eventos mediadores pelos fármacos e imunobiológicos em populações bem definidas, em termos de qualidade de vida, de ganhos terapêuticos, de prolongamento da vida ou de eventos adversos.

- Segundo Hartzema, Porta e Hugh (1998) "é a aplicação de conhecimentos epidemiológicos, métodos e raciocínio para os estudos de usos e efeitos dos medicamentos - benéficos ou adversos - na população humana".

- Segundo a Organização Mundial de Saúde (OMS) (2003a), em uma conceituação mais moderna, farmacoepidemiologia "é o estudo do uso e das RAM em um número significativo de pessoas com o objetivo de obter o uso racional, o menor custo efetivo e melhorar os resultados da saúde".

No Brasil, esse novo campo de estudo começa a ser discutido entre autoridades e profissionais de saúde na década de 1970, porém é a partir da criação da Sociedade Brasileira de Vigilância de Medicamentos (SOBRAVIME, 1991) em 1990 e de vários Centros de Informação de Medicamentos (CASTRO, 2000) que a Farmacoepidemiologia torna-se conhecida, no contexto nacional, reforçando a tese de que essa nova área do conhecimento deveria abordar a Farmacovigilância e os EUMs.

Conforme enfatizado anteriormente, os medicamentos desempenham um papel central na terapêutica desde meados do século $X X$, e sua utilização não aumentou apenas pela precisão do diagnóstico clínico, mas também devido a alterações sócio-culturais dos indivíduos. Sendo assim, os estudos Farmacoepidemiológicos são necessários no contexto atual da saúde pública, pois contribuem para detectar RAM, ineficácia do tratamento, efeitos colaterais, bem como aspectos quantitativos e qualitativos da utilização dos medicamentos, o que possibilita a realização de intervenções adequadas e oportunas nos sistemas de 
saúde e na Gestão da AF, promovendo o uso racional dos medicamentos (GUIDONI et al., 2009).

Vale salientar que a monitorização do uso dos medicamentos deve ser contínuo, dinâmico e informativo, visto que o problema ocorrido com a intoxicação por dietilenoglicol em 1930 volta a se repetir após mais de 70 anos, em 2006 no Panamá, acometendo 82 pessoas, causando 38 óbitos (MENDOZA RUIZ, OSÓRIODE-CASTRO, 2008).

\subsection{ESTUDO DE UTILIZAÇÃO DE MEDICAMENTOS}

Os EUMs são considerados uma corrente dos estudos Farmacoepidemiológicos, e apresentam visão global da utilização de medicamentos em uma sociedade. Segundo a OMS (1977), são definidos como "estudos que compreendem a comercialização, distribuição, prescrição, dispensação e uso dos medicamentos em uma sociedade, com especial enfoque em suas conseqüências médico-sanitárias, sociais e econômicas".

Segundo Lee e Bergman (2000), fica evidente na conceituação descrita acima a preocupação com fatores extrínsecos ao medicamento ou não-farmacológicos que exercem influência na utilização dos medicamentos, como os sócio-antropológicos, comportamentais e econômicos.

Os EUMs são relativamente recentes, tendo suas primeiras abordagens descritas na literatura no início da década de 1960, sendo essas publicações relacionadas com o trágico episódio da talidomida. Speiers (1960) é reconhecido como pioneiro nesses estudos, ao analisar, na Escócia, a ingestão de talidomida por mães de crianças que nasceram apresentando focomelia (CASTRO, 2000).

\subsubsection{Abordagem Multidisciplinar dos Estudos de Utilização de Medicamentos}

Para a realização dos EUMs é necessária a aplicação de três áreas do conhecimento, representadas na Figura 1, dentre as quais (HAAJER-RUSKAMP, HEMMINKI, 1993):

- Epidemiologia: necessária para auxiliar no entendimento dos amplos padrões de utilização do medicamento pela população; 
- Ciências Sociais: necessária na compreensão na percepção do indivíduo quanto ao uso do medicamento;

- Farmacologia: necessária para investigar a segurança e eficácia dos medicamentos.

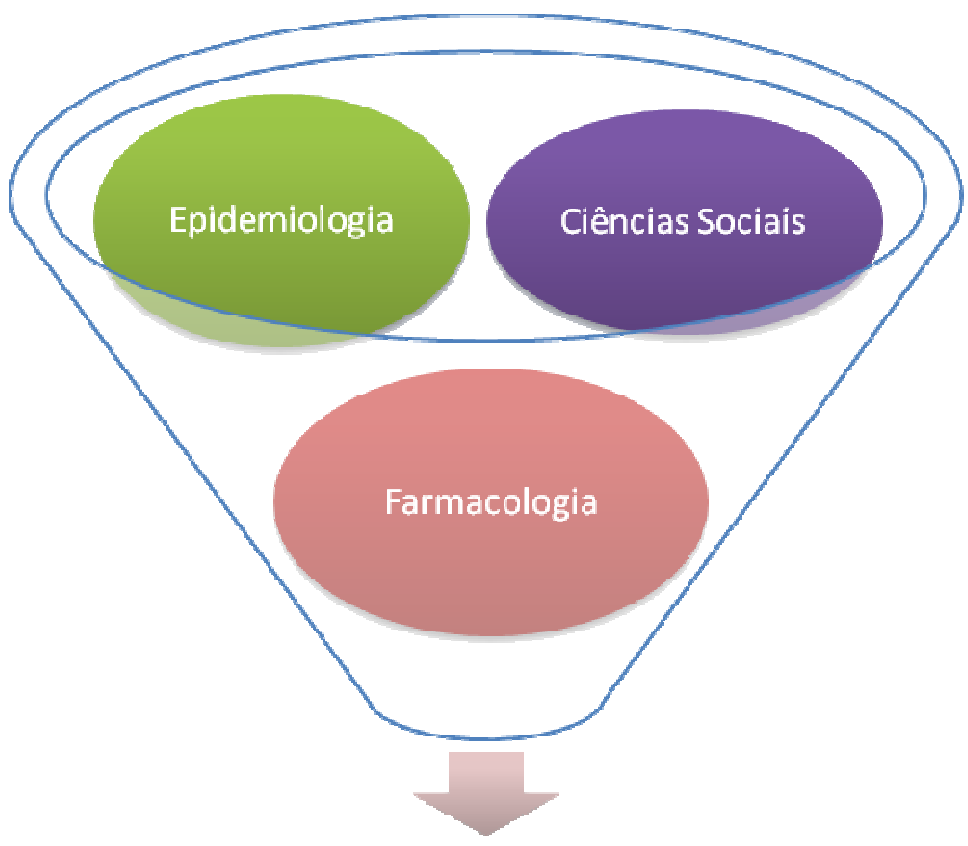

\section{Uso de Medicamentos}

Figura 1: Abordagem multidisciplinar dos Estudos de Utilização de Medicamentos.

Entretanto, os autores do presente estudo consideram que seria mais adequada a substituição da área de conhecimento farmacologia pela da terapêutica, por motivos conceituais. A farmacologia seria o estudo do uso dos medicamentos (STROM, 2000) enquanto que a terapêutica seria a aplicação dos princípios da farmacologia clínica na prescrição racional, na conduta dos estudos clínicos e na avaliação dos resultados durante a prática clínica na população (HENRY et al., 2000).

\subsubsection{Tipos de Estudos de Utilização de Medicamentos}

Os EUMs podem ser classificados em diversas categorias, sendo elas (CROZARA, 2001):

- Estudos de utilização incluindo a avaliação de oferta de medicamentos;

- Estudos quantitativos de consumo de medicamentos; 
- Estudos sobre a qualidade da prescrição;

- Estudos sobre hábitos da prescrição médica;

- Estudos de cumprimento da prescrição médica, vigilância orientada a problemas específicos, planejamento, orçamento;

- Estudos para avaliar o impacto de intervenções específicas.

De acordo com a OMS, os EUMs são importantes, pois dependendo da metodologia empregada, podem evidenciar resultados significativos, tais como (OMS, 2003a; OMS, 2004):

- Descrição de padrões de uso de medicamentos;

- Constatação de variações nos perfis terapêuticos no curso do tempo;

- Avaliação dos efeitos de medidas educativas, informativas, reguladoras;

- Estimativa do número de indivíduos expostos a medicamentos;

- Deteç̧ão de doses excessivas, uso inadequado, doses insuficientes e utilização indiscriminada dos medicamentos;

- Estimativa das necessidades de medicamentos de uma sociedade.

Com recursos de informatização e banco de dados contendo informações sobre a utilização de medicamentos, torna-se possível realizar EUMs de qualidade. Os resultados obtidos de tais estudos são fundamentais para melhorar a comunicação entre médicos e farmacêuticos, corrigindo possíveis erros de prescrição e utilização inadequada, prevenindo RAM, além de permitirem avaliar o perfil de utilização de medicamentos na população, auxiliando na gestão pública dos recursos destinados à saúde, principalmente no planejamento das políticas de $A F$, regulação sanitária (registro e fiscalização) e uso racional dos medicamentos. A presença de um sistema informatizado no município de Ribeirão Preto-SP, denominado sistema Hygia - banco de dados oficial da SMS-RP - permite a realização de EUMs.

Para a realização dos EUMs, torna-se fundamental o acesso a banco de dados que possuem informações confiáveis e completas sobre a prescrição/utilização dos medicamentos. Entretanto, nem sempre os bancos de dados disponíveis trazem informações fiéis quanto à aderência do usuário ao tratamento farmacológico prescrito, sendo esse um fator limitante para tais estudos. Porém, a utilização de sistemas informatizados apresenta vantagens como rapidez, baixo custo metodológico e a possibilidade de avaliar o consumo de medicamentos 
em número bastante significativo de indivíduos que pertencem a uma população "real", quando comparados aos estudos clínicos (clinical trial).

\subsubsection{Estudo de Utilização de Medicamentos e Diabetes mellitus}

Os EUMs são reconhecidos por serem menos onerosos, rápidos e metodologicamente aplicáveis para enfermidades que apresentam fatores específicos, como o DM, visto que o tratamento é principalmente farmacológico, sendo que os medicamentos prescritos são administrados de forma regular e contínua apenas para pacientes portadores desta enfermidade crônica. No entanto, pode-se encontrar, em alguns casos especiais, a prescrição de metformina para o tratamento da Síndrome do Ovário Policístico (HUNDAL, INZUCCHI, 2003) ou resistência à insulina.

Além disso, é reconhecido também que existe uma alta proporção de diabéticos não diagnosticados, os quais são ignorados por esses estudos, bem como os indivíduos que controlam essa patologia por medidas não-farmacológicas. Assim, o cálculo do número real de paciente que utilizam o medicamento torna-se comprometido, pois alguns estudos estimam que em 2007 nos Estados Unidos o número de portadores de DM não diagnosticados era de aproximadamente $25,0 \%$ (ADA, 2007b; ADA, 2008a).

A complexidade do manejo do DM é tão significativa que varia dentro de regiões de um mesmo país (BURGERS et al., 2002), sendo que freqüentemente ocorre a prescrição de diversas alternativas farmacológicas para pacientes com as mesmas características clínicas.

\subsection{DIABETES MELLITUS}

O DM é uma das DCNT mais prevalentes no mundo, caracterizada por hiperglicemia crônica com distúrbios no metabolismo dos carboidratos, lipídeos e proteínas, resultantes das alterações na produção e secreção e/ou no mecanismo de ação da insulina (OMS, 1999).

O DM apresenta duas formas principais, o tipo 1 (DM1), que aparece principalmente na infância ou na adolescência, devido à destruição das células beta de natureza auto-imune ou idiopática, ocasionando deficiência absoluta de insulina, 
requerendo reposição do hormônio. O tipo 2 (DM2) é a mais freqüente, e corresponde a aproximadamente $85,0-90,0 \%$ dos casos, aparece insidiosamente, principalmente em adultos (SBD, 2003; OMS, 1999), entretanto sua freqüência está aumentando em crianças e adolescentes obesos (OPAS, 2008). Essa enfermidade ocorre devido à resistência insulínica e/ou redução na secreção desse hormônio, podendo ser tratada através da utilização de dieta adequada, uso de antidiabéticos orais (ADOs), insulina ou a combinação desses (SBD, 2003; OMS, 1999).

Há outras formas menos freqüentes de DM, como a gestacional e outros tipos que ocorrem devido a defeitos genéticos funcionais das células beta e/ou na ação da insulina, induzidas por fármacos, agentes químicos ou infecções (SBD, 2003; OMS, 1999).

Além dessa classificação recomendada pela OMS, atualmente existem estudos que sugerem pacientes portadores de DM2 apresentam um alto risco de desenvolvimento da doença de Alzheimer, sugerindo que essa associação seja caracterizada como um novo tipo de DM: o Diabetes mellitus tipo 3 (WIWANITKIT, 2008).

O DM2 apresenta fatores predisponentes como: hereditariedade, obesidade, hábito alimentar inadequado, estresse e sedentarismo. Com exceção da hereditariedade, todos os outros fatores podem ser prevenidos e/ou controlados por uma dieta adequada e pela prática de atividade física regular (OMS, 2003b).

O paciente portador de DM apresenta sintomas decorrentes de insulinopenia ou déficit na ação da insulina, tais como polidipsia, poliúria, polifagia, emagrecimento, fraqueza, parestesias, turvação e diminuição da acuidade visual (OMS, 1999). A evolução da patologia sem o tratamento ou controle glicêmico ideal pode acarretar complicações agudas (cetoacidose diabética, estado hiperosmolar hiperglicêmico, hipoglicemia, acidose lática) e crônicas, tais como as microvasculares (retinopatia, nefropatia, neuropatia) e macrovasculares (amputações, disfunção sexual, doenças cardiovasculares, vasculares periféricas e cerebrovasculares) (TOSCANO, 2004).

Além disso, essa enfermidade caracteriza-se por comprometer vários sistemas orgânicos e promover alta morbi-mortalidade, além de reduzir a qualidade de vida e elevar os encargos para os sistemas de saúde, diminuindo a capacidade de trabalho e a expectativa de vida (TOSCANO, 2004). 


\subsubsection{Epidemiologia do Diabetes mellitus}

As transições demográficas, nutricionais e epidemiológicas ocorridas no século passado alteraram o perfil de risco, e as DCNT passaram a representar um importante problema de saúde pública em todo o mundo (TOSCANO, 2004).

O aumento na incidência do DM2 é atribuído a vários fatores (SARTORELI, FRANCO, 2003):

- Envelhecimento da população com fatores predisponentes;

- Alterações dietéticas, com crescente substituição dos alimentos ricos em fibra, vitaminas e minerais por produtos industrializados;

- Sedentarismo, favorecido por mudanças na estrutura de trabalho e avanços tecnológicos;

- Obesidade;

- Tabagismo, entre outros.

A prevalência mundial de DM em 1995 na população adulta era de 4,0\% (135 milhões), estimada em 5,9\% para 2007 (246 milhões) e 7,1\% para 2025 (380 milhões) (IDF, 2006). Nos países desenvolvidos ocorrerá um aumento de $42,0 \%$ nos indivíduos portadores de $\mathrm{DM}$, principalmente na faixa etária com idade mais avançada (60 a 79 anos) (KING, AUBERT, HERMAN, 1998). Na Figura 2 está representada a prevalência da enfermidade em algumas regiões do mundo.

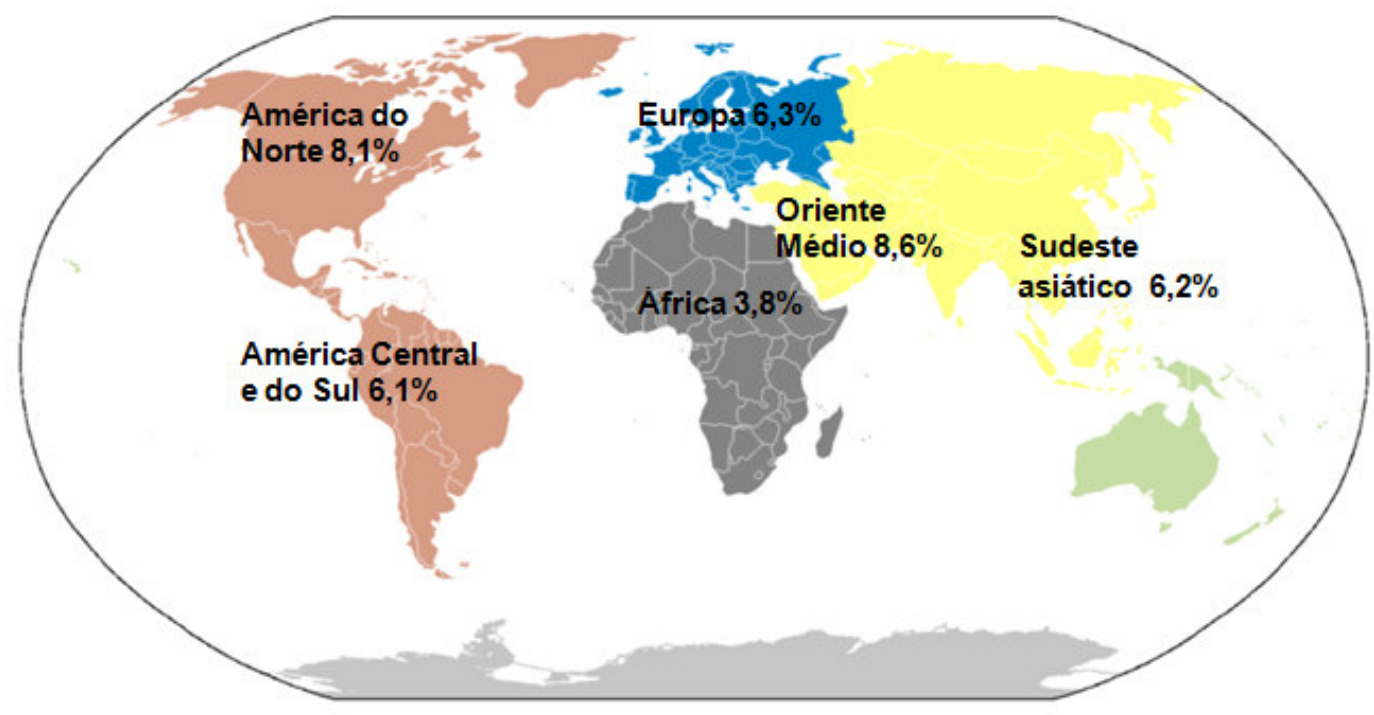

Figura 2: Prevalência de Diabetes mellitus em seis regiões mundiais no ano de 2007 (Fonte: Adaptado do $3^{\circ}$ Atlas da IDF, 2006). 
Nas Américas, o número de indivíduos com DM em 1996 situava-se em torno de 30 milhões (OPAS, ALAD, 2004), aumentando para 35 milhões em 2000, sendo estimado 45 milhões em 2010 e 64 milhões em 2025 (KING, AUBERT, HERMAN, 1998). Por volta de 2025, calcula-se que a ocorrência do DM na região das Américas Central e do Sul seja tão elevada (9,3\%) quanto à norte-americana $(9,7 \%)$. Estudos recentes demonstram que a cada ano, 7 milhões de pessoas desenvolvem o DM, ou seja, um caso novo a cada 5 segundos (IDF, 2006). Apenas nos Estados Unidos surgem 1 milhão de novos casos por ano (ADA, 2008a).

O Brasil possuía 4,9 milhões de portadores de DM em 1995, sendo estimando para 2007 e 2025, respectivamente 6,9 e 17,6 milhões, ou seja, aproximadamente de $8,0 \%$ a $20,0 \%$ da população (IDF, 2006).

O estágio de pré-diabetes, conhecido como Intolerância a Glicose (IGT), tem merecido destaque, pois os usuários com IGT têm risco significativo de desenvolver DM2. Estima-se que aproximadamente 308 milhões de pessoas apresentaram quadro de IGT em 2007 e projeta-se para 2025 uma prevalência de 8,1\%, ou seja, 418 milhões de pessoas (IDF, 2006), fato que aumentará significativamente a prevalência de DM e que é negligenciado pelos países.

Uma situação preocupante é observada quando o assunto é DM na infância, pois essa patologia é crônica, sendo que dados recentes indicam que uma em cada três crianças nascidas nos Estados Unidos desenvolverá diabetes em algum momento da vida. Quando essa doença é diagnosticada nos jovens, a expectativa de vida é reduzida, em média, de dez a vinte anos (IDF, 2006).

A maioria dos países latino-americanos não desenvolveu sistema de vigilância epidemiológica para as DCNT na população, em particular a DM (SARTORELLI, FRANCO, 2003), acarretando assim, um impacto negativo sobre a qualidade de vida e uma sobrecarga ao sistema de saúde (KING, AUBERT, HERMAN, 1998), desestabilizando toda rede assistencial, inclusive o cuidado e atenção para outras enfermidades.

\subsubsection{Custo do Diabetes mellitus}

Os custos para os cuidados de uma enfermidade crônica são elevados, sendo que muitas vezes o valor gasto compreende a redução dos dias trabalhados e da produtividade no trabalho e a aposentadoria precoce. Entretanto, nesses casos os encargos ultrapassam a esfera financeira, relacionando também custos sociais e 
humanísticos, levando a redução da qualidade de vida e, muitas vezes, a problemas que interferem psicologicamente na saúde do usuário (GUIDONI et al., 2009).

Mundialmente, os custos diretos para o atendimento aos portadores do DM variam de 2,5 a 15,0\% dos investimentos nacionais em saúde, dependendo da prevalência local e da complexidade do tratamento disponível. Portadores de DM consomem pelo menos duas vezes mais recursos para o cuidado com a saúde, quando comparado aos não-diabéticos. (OPAS, ALAD, 2004).

Os indivíduos portadores de DM, quando comparados aos indivíduos sem diagnóstico dessa patologia, utilizam mais recursos hospitalares, como atendimento ambulatorial, visitas médicas, consulta a outros profissionais de saúde, atendimentos de urgência e emergência e prescrição de medicamentos e suprimentos (ADA, 2008a).

O custo anual total dos pacientes portadores de DM hospitalizados foi de seis a sete vezes superiores quando comparados aos portadores não hospitalizados (CAPORALE, CALVO, GAGLIARDINO, 2006), demonstrando que a hospitalização é um dos maiores fatores de elevação do custo no tratamento da enfermidade.

$\mathrm{Na}$ Europa, o custo médio para o cuidado do paciente portador de DM varia de $€ 1,3$ mil na Espanha $a € 3,6$ mil na Alemanha. Além disso, pode-se observar que o montante investido no cuidado aos portadores de DM alcançou 7,4\% do orçamento com saúde na Itália; 6,7\% na Bélgica; 6,3\% na Alemanha; 4,4\% na Espanha durante o ano 1998 (PASSA, 2002).

Nos Estados Unidos, no ano de 1997, o gasto com o cuidado prestado aos pacientes portadores de DM foi 2,3 vezes maior quando comparado aos pacientes que não possuíam o diagnóstico desta patologia. Nesse mesmo país, a estimativa total de custo para tratamento do DM em 2007 foi de US\$ 174 bilhões, incluindo o custo direto de US\$ 116 bilhões em gastos médicos e o custo indireto orçado em US\$ 58 bilhões devido à redução da produtividade nacional (ADA, 2008a).

Os custos diretos atribuídos ao DM incluem US\$27 bilhões para o cuidado do tratamento, US\$ 31 bilhões nos custos médicos em geral e US\$ 58 bilhões para os tratamentos das complicações atribuídas a essa enfermidade. A hospitalização corresponde a 50,0\% dos custos totais para o cuidado do DM, a medicação e suprimentos para o tratamento alcançam 12,0\%, aquisições de medicamentos para tratamento das complicações somam $11,0 \%$ e as consultas médicas atingem a 9,0\% (ADA, 2008a). Observa-se que os gastos com a hospitalização e as complicações 
são os fatores de maior impacto nos custos do cuidado aos pacientes portadores de DM, mesmo sabendo que as causas para ocorrência dessas complicações são, muitas vezes, modificáveis e preveníveis.

Aproximadamente $50,0 \%$ dos custos indiretos são causados por óbitos dos pacientes portadores de DM enquanto que 35,0\% são devido à redução na produtividade no trabalho, ocasionadas pela ausência de 14 dias em média ao trabalho no ano (ADA, 2008a).

No Brasil, o Sistema de Informação Hospitalar do SUS demonstra que as internações referentes ao DM representaram gastos de aproximadamente $R \$ 39$ milhões no SUS em 2000, devido à alta taxa de permanência hospitalar decorrente da severidade das complicações, que demandam ainda a realização de procedimentos de alta complexidade (MARIN et al., 2003), os quais oneram o sistema de maneira significativa.

Recentemente no país tem-se observado um crescente número nas hospitalizações devido aos agravamentos pertinentes ao DM. Na região de Ribeirão Preto-SP, no período de 1988 a 1997, registrou-se um aumento de 14,3\% nas hospitalizações, sendo que desse total $33,6 \%$ tinham o DM como causa principal e 53,9\% mencionavam o DM como causa secundária (FRANCO, ROCHA, 2002).

Num estudo realizado de janeiro a dezembro de 2004, num hospital privado de João Pessoa-PB, cerca de 7,0\% dos indivíduos hospitalizados eram portadores de DM. O tempo médio de internação foi de 9,1 dias e o custo médio de $R \$ 12$ mil (MODESTO-FILHO et al., 2007).

Do total de gastos hospitalares do governo brasileiro no ano de $2002, R \$ 7,5$ bilhões $(69,1 \%)$ foram destinados aos pacientes portadores de DCNT (MS, 2005). Devido à prevalência e a severidade, o portador de DM gera grande impacto econômico e social, devido à alta freqüência de complicações agudas e crônicas, hospitalizações, baixa produtividade pessoal, aposentadoria precoce, invalidez ou morte prematura (BARCELÓ et al., 2001). Além disso, essa patologia está associada à dor, ansiedade, inconveniência e menor qualidade de vida dos pacientes e familiares (OPAS, 2005), principalmente quando não controlada.

Indivíduos portadores de DM apresentam expectativa de vida inferior, aproximadamente 10 anos, quando comparados a indivíduos sem o diagnóstico dessa enfermidade (IDF, 2006). A cada ano, cerca de 4 milhões de mortes prematuras são relacionadas ao DM e suas complicações, o que representa $9,0 \%$ da 
mortalidade mundial total (IDF, 2006; MS, 2006a). Entretanto, sabe-se que o subregistro da mortalidade do DM é conhecido, sendo que muitas vezes o motivo do óbito se deve a complicações do DM, como nefropatia ou doença cardiovascular (BARCELÓ et al., 2003).

Na América Latina e Caribe o DM ocupou o 4ํlugar como causa de morte no ano de 2001, o que representou 5,0\% do total de óbitos, sendo que no México foi a primeira causa de morte na população total em 2002, com 12,8\% dos óbitos (OPAS, 2007).

O DM é a 5ำ causa de morte nos Estados Unidos, tendo efeito significativo no sistema de saúde (HOGAN, DALL, NIKOLOV, 2003). É a causa primária em 33,0\% dos casos de insuficiência renal que evoluem para diálise ou transplante (PERNEGER et al., 1994). Cerca de 50,0\% das amputações de membros inferiores nos Estados Unidos ocorrem em indivíduos portadores de DM (HUMPHREY et al., 1994). O risco de desenvolver doenças coronarianas e acidentes vasculares cerebrais é de duas a quatro vezes superiores nesses pacientes, quando comparados com não-diabéticos da mesma idade e riscos similares de doença cardiovascular (MORRISH et al., 2001).

O risco relativo de morte por doenças isquêmicas do coração é três vezes maior em pacientes portadores de DM quando comparado aos usuários sem a enfermidade, além disso, o risco relativo de morte por doenças renais é cinco vezes maior nesses mesmos pacientes (GU, COWIE, HARRIS, 1998).

A freqüência e a severidade das complicações do DM não controlada têm exigido investimentos cada vez maiores. Pacientes com complicações macro e microvasculares apresentam gasto três vezes maior que pacientes sem complicações (BRANDLE et al., 2003; HENRIKSSON, AGARDH, BERNE, 2000). A despesa média anual do governo norte-americano, em 2002, foi de US\$13 mil para portadores de DM e apenas US\$2,6 mil para indivíduos não portadores de DM (HOGAN, DALL, NIKOLOV, 2003).

O custo do paciente aumenta com o decorrer do tempo, especialmente devido à presença de complicações tardias. O custo anual do tratamento de indivíduos portadores de DM na Suécia por até cinco anos foi em média de $€ 2,2$ mil, enquanto que indivíduos com diagnóstico de DM superior a 10 anos apresentaram custo de $€$ 3,3 mil. Os agravamentos foram decisivos no aumento de gastos, pois o custo paciente/ano sem complicações foi de $€ 1,7 \mathrm{mil}$, enquanto que para aqueles que 
apresentaram agravamentos macro e microvasculares o custo superou $€ 5,0$ mil, sendo que $75,0 \%$ deste montante foram destinados às hospitalizações (HENRIKSSON, AGARDH, BERNE, 2000).

O aumento nos custos de tratamento do portador de DM tem várias causas, tais como, novos casos diagnosticados, prevalência da doença, inflação nos preços dos cuidados médicos, ampliação na utilização de serviços médicos desses indivíduos, desenvolvimento de novas tecnologias para o tratamento e aumento no número e na gravidade das complicações ao longo do tempo (OPAS, 2005).

No Brasil, o DM recebe atenção especial por parte do sistema de saúde, porém, não existem dados conclusivos sobre a contribuição relativa dos gastos com os portadores dessa enfermidade. Um estudo do MS, em 2002, demonstrou que as DCNT são responsáveis pelas maiores proporções de anos de vida perdidos por morte prematura $(59,0 \%)$, por anos de vida vividos com incapacidade $(74,7 \%)$ e por anos de vida perdidos ajustados por incapacidade (66,3\%) (SCHRAMM et al., 2004). A expectativa média de vida é reduzida em 15 anos para portadores de DM1 e 7 anos para o DM2 (MS, 2006a).

O número de óbitos devido ao DM aumentou em 26,0\% de 1995 a 1999, alcançando uma taxa de 22 óbitos para 100 mil habitantes no ano de 2003 (MELLOJORGE, GOTLIEB, LAURENTI, 2001; MS, 2005). A tendência de aumento na incidência de DM, devido à elevação da expectativa de vida, torna a enfermidade um grave problema de saúde pública (SBD, 2003).

A OMS estimou que 35 milhões de pessoas morreram por DCNT no mundo em 2005, o que representou $60,0 \%$ de todas as mortes, sendo que desse montante 1,1 milhão $(3,1 \%)$ de óbitos foram referidos à presença do DM. Além desses problemas sanitários, as repercussões econômicas são enormes, estimando-se que a perda de renda nacional devido às doenças cardiovasculares e DM atingirão, nos próximos dez anos, US $\$ 49,2$ bilhões no Brasil, alcançando 0,5\% do PIB em 2015 (OMS, 2005). Esse custo será responsável por onerar a renda nacional, interferindo diretamente no cuidado de outras enfermidades, visto que ocorrerá uma diminuição no aporte financeiro destinado a elas (GUIDONI et al., 2009).

A necessidade de reformulação dos serviços prestados aos portadores de DM é observada quando se analisa os resultados encontrados por Caporale, Calvo e Gagliardino (2006), que observaram que o aumento no número de consultas 
médicas não foi eficaz para prevenir as hospitalizações ou re-hospitalizacões dos portadores de DM.

Os três pilares fundamentais na assistência global do paciente portador de DM são: controle glicêmico rígido (dieta/estilo de vida, exercício físico, medicação), tratamento de distúrbios associados (dislipidemia, hipertensão, obesidade, coronariopatia) e pesquisa e/ou tratamento das complicações da enfermidade (retinopatia, doença cardiovascular, nefropatia, neuropatia, outras complicações) (ADA, 2008b). Sendo assim, o cuidado prestado ao paciente portador de DM transcende o descontrole glicêmico característico da enfermidade e passa a representar uma enormidade de variáveis necessárias de serem controladas.

A melhora no controle do DM pode ser alcançada por meio do tratamento não-medicamentoso ou medicamentoso. O primeiro tem como finalidade primária retardar a implantação da doença, e quando já implantada evitar ou adiar o tratamento farmacológico ou impedir o aumento do número de medicamentos necessários para o controle da doença. O segundo deve ser introduzido quando não obtiver sucesso com o primeiro. Em ambos é necessária a compreensão e adesão do portador, pois se trata de doença crônica, sendo que seu controle baseia-se na redução do número de agravamentos.

\subsubsection{Tratamento não-farmacológico do Diabetes mellitus}

Para o tratamento do portador de DM é imprescindível à vinculação do paciente às unidades de atendimento, garantindo o diagnóstico e acesso às formas de tratamento, aliado ao atendimento por profissionais capacitados, uma vez que o diagnóstico e controle evitam complicações ou, ao menos, retardam a progressão das já existentes. Além disso, o maior contato com o serviço de saúde promove maior adesão ao tratamento (MS, 2002a).

A terapêutica medicamentosa é a principal forma de tratamento dos pacientes portadores de DM. Com o passar dos anos, o diabético apresenta agravamento no controle da enfermidade, principalmente se não tratada adequadamente. Entretanto, a terapêutica não-farmacológica deve ser realizada por toda a vida e, muitas vezes, quando introduzida logo após o diagnóstico, retarda 0 aparecimento das complicações e também o início do tratamento medicamentoso (ADA, 2007a)

O tratamento não-farmacológico dispensado ao portador de DM e outras DCNT como HAS, obesidade e dislipidemias incluem as seguintes estratégias: 
educação, modificações do estilo de vida, aumento da atividade física, reorganização dos hábitos alimentares, redução de peso e diminuição ou abandono de alguns vícios prejudiciais à saúde, como fumo e álcool (ADA, 2007a; SBD, 2003).

A importância da prevenção primária do DM2 no Brasil tem sido enfatizada por diversos epidemiologistas (SARTORELLI, FRANCO, 2003) fazendo-se necessário o desenvolvimento de programas eficazes e viáveis ao SUS, tais como prevenção primária, controle da incidência e prevenção secundária das complicações metabólicas da enfermidade (SARTORELLI, FRANCO, CARDOSO, 2006). Além disso, o maior contato com serviços de saúde tem efeito protetor, estando relacionado a uma probabilidade menor do indivíduo vir a óbito por agravamentos relacionados ao DM (DERI, 1995).

Assim, é possível afirmar que a mudança dos hábitos possa prevenir ou retardar o desenvolvimento de complicações, reduzindo substancialmente o custo dessa enfermidade (DPP, 2002). No entanto, a mudança no estilo de vida não é tão simples e depende de fatores psicológicos, sociais e econômicos (MOLENAFERNANDES et al., 2005), sendo importante que o cuidado prestado a esse paciente seja conduzido por uma equipe interdisciplinar.

Entretanto, durante o curso da doença, dificilmente o portador de DM não necessitará utilizar medicamentos para o tratamento, pois na evolução dessa enfermidade ocorre a redução da produção e secreção de insulina das células beta do pâncreas. Logo, o tratamento medicamentoso deve ser realizado conjuntamente com o tratamento não-farmacológico.

\subsubsection{Tratamento farmacológico do Diabetes mellitus}

A maioria dos portadores do DM apresenta comorbidades, tais como: obesidade, HAS e dislipidemia. Portanto, para o cuidado dessa enfermidade tornase necessária uma abordagem interdisciplinar, colaborativa e integrada da equipe de saúde, que deve estimular o paciente a executar um papel ativo no auto-cuidado (ARAÚJO et al., 1999).

Os portadores de DM1 geralmente são diagnosticados na infância, pois na maioria das vezes a destruição das células beta do pâncreas por auto-imunidade ou devido a causas idiopáticas ocorre principalmente nesta fase da vida. Dessa forma, pode-se se afirmar que a enfermidade se inicia de forma abrupta e necessita de tratamento imediato com insulina, além de uma adequação alimentar. A insulina é 
até hoje a única opção medicamentosa para o tratamento do DM1 (SBD, 2003; OMS, 1999).

Além disso, a insulina foi o primeiro medicamento descoberto para o tratamento do DM, em 1922 por Banting e Best, sendo que somente após 32 anos, em 1954, foram comprovados os efeitos clínicos dos primeiros medicamentos orais para tratamento da enfermidade, ou seja, as sulfoniluréias (RENDELL, 2004).

Com a descoberta dos efeitos hipoglicemiantes das sulfoniluréias em 1954, o tratamento farmacológico do DM2 sofreu alterações significativas, pois a partir desse momento, a utilização de medicamentos orais tornou-se a principal ferramenta no cuidado desses pacientes, sendo que também em meados da década de 1950 ocorreu a descoberta de uma nova classe de medicamentos para o tratamento do DM2, as biguanidas (RENDELL, 2004).

Entretanto, os primeiros representantes desta nova classe, fenformina e buformina, apresentaram reações adversas graves, tais como acidose lática, fazendo com que esses medicamentos fossem temporariamente proscritos (HUNDAL, INZUCCHI, 2003). Porém, com a descoberta da meftormina, uma nova biguanida, considerada mais segura, ocorreu crescimento acentuado na prescrição desse medicamento, sendo que hoje, ao lado das sulfoniluréias, constituem as principais opções terapêuticas prescritas.

O objetivo da terapêutica farmacológica do DM2 é alcançar o controle glicêmico satisfatório em pacientes que não o conseguiram através de medidas nãofarmacológicas. Em relação ao tratamento medicamentoso, existem no momento diversas opções que podem ser utilizadas isoladamente ou em associações (SBD, 2003). Os ADOs são substâncias que, quando ingeridas, têm finalidade de diminuir a glicemia e mantê-la normal (jejum < $100 \mathrm{mg} / \mathrm{dl}$ e pós-prandial < $140 \mathrm{mg} / \mathrm{dl}$ ) (ADA, 2008b).

Os ADOs podem ser classificados em duas categorias: os que não aumentam a secreção de insulina - anti-hiperglicemiante e os que aumentam a secreção de insulina - hipoglicemiante. A insulina pode ser utilizada no tratamento do DM2 dependendo da característica fisiopatológica apresentada pelo paciente (SBD, 2003).

Os medicamentos que não aumentam a secreção de insulina quando usados em monoterapia não estão relacionados ao aparecimento de hipoglicemia e, portanto, podem ser utilizados com segurança desde o início da enfermidade. Assim 
fazem parte deste grupo: a metformina (biguanida), a rosiglitazona e pioglitazona (tiazolidinedionas ou glitazonas) e a acarbose (inibidor da alfa-glicosidase). A metformina tem sua maior ação anti-hiperglicemiante na diminuição da produção hepática de glicose, com uma ação sensibilizadora periférica, sendo o fármaco de escolha no paciente obeso portador de DM2 (SBD, 2003; UKPDS, 1998a). As glitazonas atuam principalmente na insulino-resistência periférica no músculo, célula adiposa e hepatócito, enquanto que a acarbose reduz a velocidade de absorção intestinal de glicose, agindo principalmente na glicemia pós-prandial (SBD, 2003).

Os medicamentos que aumentam a oferta de insulina são conhecidos como os secretagogos de insulina, sendo eles as sulfoniluréias que desenvolvem uma ação hipoglicemiante mais prolongada (clorpropamida, glibenclamida, glicazida, glipizida, e glimepirida) e as glinidas, com menor tempo de ação, cobrindo principalmente o período pós-prandial, como a nateglinida e a repaglinida (SBD, 2003; TURNER et al., 1999; UKPDS, 1998a).

Qualquer que seja o esquema terapêutico, o controle glicêmico é de fundamental importância na prevenção ou redução das complicações micro e macrovasculares. O Diabetes Control and Complications Trial, um estudo prospectivo multicêntrico sobre as diversas formas de tratamento com insulina no DM1 (DCCT, 1996) e, mais recentemente, o United Kingdom Prospective Diabetes Study (UKPDS) que avaliou o tratamento farmacológico no DM2, mostraram a fundamental importância do controle glicêmico na prevenção ou redução das complicações micro e macrovasculares. O UKPDS mostrou que o desenvolvimento de complicações microvasculares no DM2 foi reduzido quando prescrito sulfoniluréia, metformina ou insulina, entretanto apenas pacientes em uso de metformina apresentaram diminuição significativa das complicações macrovasculares (TURNER, 1998).

No entanto, independente do tipo de tratamento, ocorre uma piora progressiva da função da célula beta pancreática, sendo que após nove anos de tratamento, $75,0 \%$ dos pacientes necessitam associar diversas terapias para manutenção do controle glicêmico (TURNER, 1998). Este quadro progressivo é uma evolução natural da enfermidade, entretanto pode ser acelerado quando o esquema terapêutico prescrito não é cumprido, sendo que a adesão ao tratamento farmacológico deve ser estimulada freqüentemente nos pacientes portadores de DCNT como o DM2, pois segundo estimativas o cumprimento integral da prescrição 
médica por esses indivíduos situa-se em torno de 50,0\% (DUARTE-RAMOS, CABRITA, 2006).

Levando-se em consideração as fases de progressão do DM2, conforme representado na Figura 3, pode-se observar que a Fase 1 geralmente ocorre com predomínio de obesidade e resistência insulínica e os medicamentos mais indicados são sensibilizadores de insulina. Na Fase 2, com diminuição significativa de secreção de insulina, é indicada associação de um secretagogo de insulina na maioria dos casos. Na Fase 3, com a progressão da perda da capacidade de secreção, é necessário associar aos agentes orais a aplicação de insulina de depósito ao deitar. Por fim, na Fase 4, quando predomina a insulinoterapia, o paciente deve receber pelo menos duas aplicações de depósito (antes do desjejum e jantar ou dormir), isoladas ou combinadas com uma insulina rápida ou ultra-rápida. Nessa Fase, um agente oral sensibilizador combinado no tratamento pode reduzir as doses de insulina e auxiliar na melhora do controle metabólico (UKPDS, 1995).

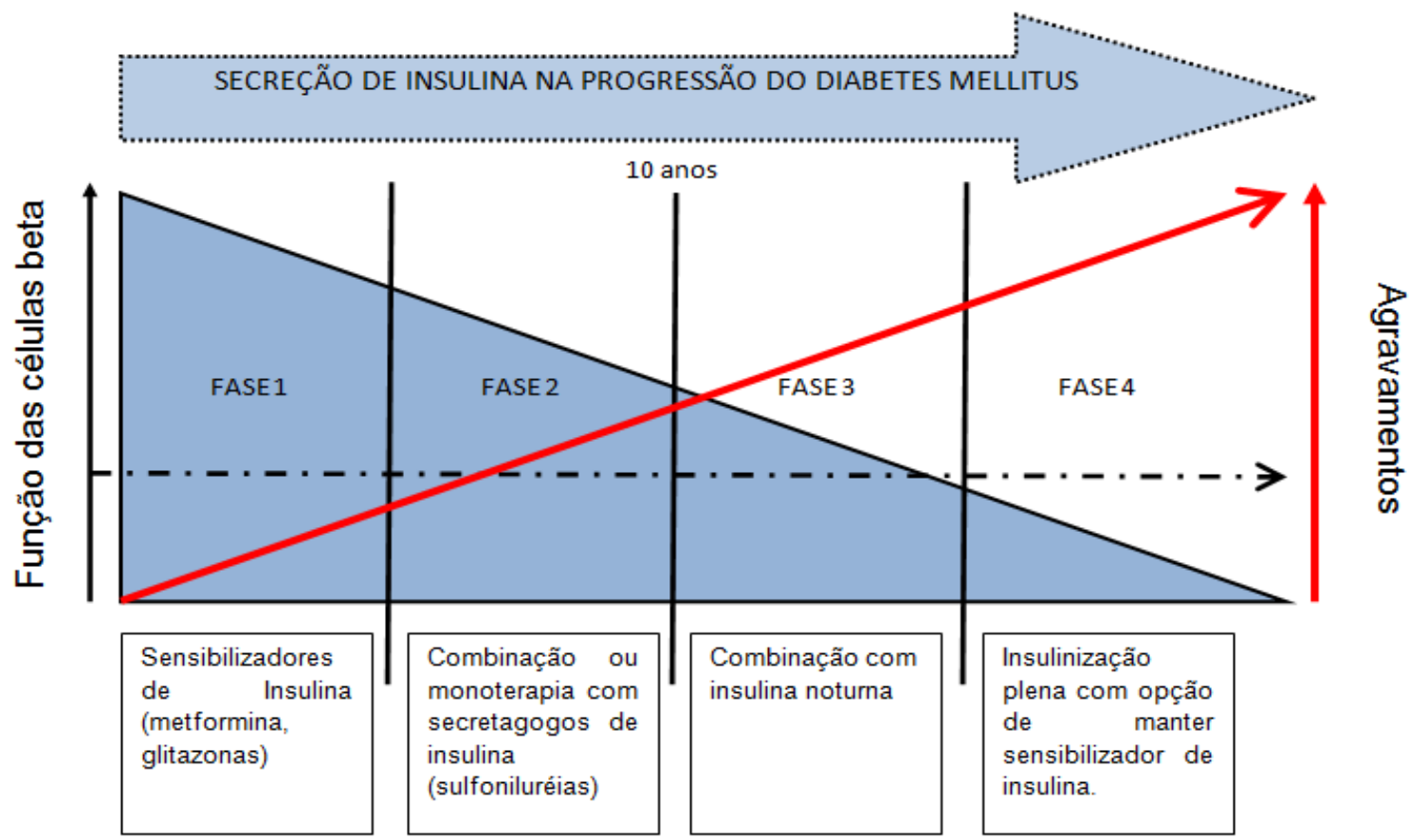

Figura 3: Algoritmo terapêutico para o manejo do Diabetes mellitus tipo 2 segundo a fase de evolução da doença (Adaptado de Monnier et al., 2005).

$\mathrm{Na}$ escolha do medicamento ADOs para o tratamento do DM deve ser observado os valores das glicemias de jejum e pós-prandial e da hemoglobina glicosilada, o peso e idade do paciente, a presença de complicações, outros 
transtornos metabólicos e doenças associadas e as possíveis interações com os medicamentos, reações adversas e as contra-indicações (SBD, 2006).

Abaixo, na Tabela 1, estão detalhados os três medicamentos ADOs disponibilizados pela SMS-RP utilizados para o tratamento do DM2.

Tabela 1 - Características terapêuticas dos antidiabéticos orais disponibilizados pela SMS-RP

\begin{tabular}{|c|c|c|c|c|c|c|c|}
\hline & Medicamento & $\begin{array}{l}\text { Mecanismo } \\
\text { de ação }\end{array}$ & $\begin{array}{c}\text { Dose } \\
\text { (mg/dia) }\end{array}$ & $\begin{array}{l}\text { Duração da } \\
\text { ação }\end{array}$ & $\begin{array}{l}\text { Atividade } \\
\text { dos } \\
\text { metabólitos }\end{array}$ & $\begin{array}{c}\text { Via de } \\
\text { eliminação }\end{array}$ & $\begin{array}{l}\text { Contra- } \\
\text { indicação }\end{array}$ \\
\hline \multirow{2}{*}{ 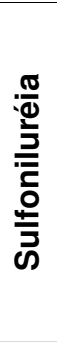 } & GLI & $\begin{array}{l}\text { - Aumento da } \\
\text { secreção de } \\
\text { insulina }\end{array}$ & $2,5-15,0$ & $\begin{array}{c}\text { Intermediária } \\
\text { a longa }^{\mathrm{a}}\end{array}$ & Ativo & $\begin{array}{l}\text { Bile } \approx \\
50,0 \%\end{array}$ & $\begin{array}{c}\text { Gravidez, } \\
\text { IR e IH }\end{array}$ \\
\hline & GCZ & $\begin{array}{l}\text { - Aumento da } \\
\text { secreção de } \\
\text { insulina }\end{array}$ & $80-320$ & Intermediária ${ }^{a}$ & Inativo & $\begin{array}{l}\text { Urina } \approx \\
65,0 \%\end{array}$ & $\begin{array}{c}\text { Gravidez, } \\
\text { IR e IH }\end{array}$ \\
\hline $\begin{array}{l}\frac{\pi}{0} \\
\frac{0}{2} \\
\frac{\pi}{0} \\
\frac{0}{0}\end{array}$ & MET & $\begin{array}{l}\text { Reduz a } \\
\text { produção } \\
\text { hepática de } \\
\text { glicose; } \\
\text { sensibilizador } \\
\text { da ação } \\
\text { insulínica }\end{array}$ & $\begin{array}{l}500- \\
2550\end{array}$ & - & $\begin{array}{l}\text { Não possui } \\
\text { metabólitos }\end{array}$ & $\begin{array}{l}\text { Urina } \approx \\
90,0 \%\end{array}$ & $\begin{array}{c}\text { Gravidez, } \\
\text { IR, IH, IC, } \\
\text { IP, } \\
\text { acidose } \\
\text { grave, } \\
\text { alcoolismo }\end{array}$ \\
\hline & $\begin{array}{l}\text { a >24h; intern } \\
\text { alibenclamida } \\
\text { Medicamentc } \\
\text { suficiência rer } \\
\text { : Hundal e Inz }\end{array}$ & $\begin{array}{l}\text { ediário 12-24h; } \\
\text { GCZ: Glicazida }\end{array}$ & $\begin{array}{l}\text { irta }<12 \mathrm{~h} . \\
\text { MET: Mett }\end{array}$ & $\begin{array}{l}\text { rmina. } \\
\text { IC: Insuficiênc }\end{array}$ & rardín & Incuficiô & pulmonar. \\
\hline
\end{tabular}

Portanto, o principal objetivo do tratamento farmacológico do paciente portador de DM é a manutenção de níveis glicêmicos e de hemoglobina glicosilada, melhorando a qualidade de vida e diminuindo a ocorrência de complicações. Além disso, torna-se fundamental nesses pacientes o tratamento das comorbidades, tais como HAS, obesidade e dislipidemia. Por isso, o tratamento do paciente portador de DM torna-se complexo, envolvendo uma série de fatores e de morbidades.

\subsubsection{Tratamento das comorbidades associadas ao Diabetes mellitus}

A primeira etapa do tratamento das comorbidades (HAS, obesidade e dislipidemia) no paciente diabético deve ser conduzida da mesma forma para os não-diabéticos, iniciando por meio de medidas não-farmacológicas (ADA, 2004), e quando necessário a introdução do tratamento medicamentoso. 
A HAS é comum em pacientes portadores de DM, com uma prevalência de 40,0-60,0\% na faixa etária de 45 a 75 anos (UKPDS, 1998b). A sua presença acarreta aumento do risco cardiovascular (duas vezes mais quando comparado aos hipertensos não-diabéticos) e pode promover ou acelerar as lesões micro e macrovasculares do paciente portador de DM2 (ADA, 2004), tais como doença renal e retinopatia diabética.

Os pacientes portadores de DM beneficiam-se do controle da pressão arterial pela redução marcada e evidente de todos os indicadores de morbidades e mortalidades globais, cardiovasculares e renais associados à diminuição do nível pressórico a valores inferiores a 130/80 $\mathrm{mm} \mathrm{Hg}$ e, particularmente, menores que 120/80 mm Hg para pacientes com proteinúria ou insuficiência renal (ADA, 2008b; UKPDS, 1998b).

Os medicamentos que produzem inibição do sistema renina-angiotensina aldosterona, como os inibidores da enzima conversora de angiotensina (iECA) e os antagonistas dos receptores de angiotensina II (ALVARENGA, 2005) são considerados de primeira escolha no tratamento da maioria dos pacientes portadores de DM e HAS, pois não apresentam efeitos no metabolismo dos glicídios e lipídeos e podem reduzir a progressão da nefropatia diabética (ADA, 2004; JAWAD, GREGORY, 2006).

Os diuréticos tiazídicos podem ser utilizados em baixas doses para o tratamento de alguns pacientes (ALVARENGA, 2005; GUS, FUCHS, 2006; ALLHAT, 2002), pois podem interferir na secreção de insulina, acarretando em aumento da glicemia. Os beta-bloqueadores e os antagonistas dos canais de cálcio são úteis no tratamento de hipertensos diabéticos com doença coronária e/ou insuficiência cardíaca (ALVARENGA, 2005). Entretanto, os beta-bloqueadores podem apresentar efeitos metabólicos adversos, como deteriorização do metabolismo glicolipídico, diminuição da sensibilidade insulínica, além de mascarar e prolongar a hipoglicemia (GRESS et al., 2000).

A associação de dois ou mais fármacos é uma atitude adequada e necessária para se alcançar o controle da pressão arterial nos pacientes portadores de DM e na maioria das vezes é utilizada no início da terapia (JAWAD, GREGORY, 2006).

Outra comorbidade altamente associada ao DM é a obesidade, que juntamente com outras enfermidades eleva o risco de ocorrência da Síndrome Metabólica (GRUNDY et al., 2004) sendo um importante fator de risco na evolução 
do DM, HAS e dislipidemia (GOMES et al., 2006). A prevalência de obesidade nos pacientes diabéticos foi três vezes maior do que a observada na população brasileira em geral de acordo com os dados do IBGE (IBGE, 2007).

Pequenas reduções de peso $(5,0$ a $10,0 \%)$ se associam à melhora significativa nos níveis pressóricos, nos índices de controle metabólico, na sensibilidade à insulina e reduzem a morbi-mortalidade relacionada ao DM (SBD, 2003). Infelizmente, a perda de peso é difícil de ser alcançada apenas com tratamento não-farmacológico (MILES et al., 2002), principalmente em paciente portadores de $\mathrm{DM}$, pois o próprio tratamento da enfermidade com sulfoniluréia e insulina pode ocasionar aumento de peso.

A introdução do tratamento medicamentoso com agentes antiobesidade deve seguir uma racionalidade (SPITZ, HEYMSFELD, BRANK, 1995), sendo que os medicamentos disponíveis no mercado são classificados como anorexígenos (femproporex, dietilpropiona e mazindol), sacietógenos (fluoxetina, sertralina e sibutramina) e redutores da absorção intestinal de gorduras (orlistat). Vale salientar que dentro do grupo de medicamentos sacietógenos o único considerado como agente antiobesidade é a sibutramina (OMS, 1998). Em alguns casos, pacientes diabéticos com IMC > 35kg/ $\mathrm{m}^{2}$ e sem melhora nos níveis glicêmicos podem recorrer à cirurgia bariátrica (CONSENSO LATINO-AMERICANO DE OBESIDADE, 1998).

A dislipidemia constitui outra comorbidade associada ao DM, sendo que portadores de DM2 possuem de duas a quatro vezes mais risco de desenvolverem doenças cardiovasculares (DCV) quando comparados a pacientes não-diabéticos. As DCV são responsáveis por $75,0 \%$ das mortes de indivíduos com DM2 (SBD, 2006).

Segundo recomendações da American Diabetes Association (ADA), a ordem de prioridades para o tratamento da dislipidemia diabética é (ADA, 2008b):

- Redução da Lipoproteína de baixa densidade (LDL-C) a valores menores que $100 \mathrm{mg} / \mathrm{dl}$;

- Elevação da Lipoproteína de alta densidade (HDL-c) para valores acima de $40 \mathrm{mg} / \mathrm{dl}$;

- Diminuição dos Triglicerídeos a valores abaixo de $150 \mathrm{mg} / \mathrm{dl}$.

O tratamento da dislipidemia é extremamente importante em pacientes diabéticos, devido à elevada prevalência de doença coronariana. Os medicamentos de primeira escolha são as estatinas e os fibratos. O primeiro deve ser introduzido 
no tratamento quando o LDL-c for superior a $130 \mathrm{mg} / \mathrm{dL}$ e os derivados do ácido fíbrico devem ser utilizados quando o valor de triglicerídeos superar os $500 \mathrm{mg} / \mathrm{dL}$ (ADA, 2008b).

Assim, conforme exposto anteriormente, percebe-se que o DM é uma enfermidade de tratamento complexo, associado a várias comorbidades, necessitando de uma abordagem interdisciplinar e melhor atenção dos sistemas de saúde, sendo que se tornou uma das doenças mais prevalentes em todo o mundo, podendo ser considerada a epidemia do século XXI.

Segundo a OMS, ainda que responsáveis pela maioria das mortes, as DCNT permanecem negligenciadas, não sendo contempladas na agenda das prioridades dos governos em todo o mundo (OPAS, 2005). As DCNT apresentam um custo elevado para o SUS e quando não prevenidas e gerenciadas adequadamente demandam uma assistência médica de custo crescente, em razão da permanente e necessária incorporação tecnológica para o seu controle (MALTA et al., 2006).

Por conseguinte, os EUMs são uma ferramenta útil na avaliação da utilização de medicamentos ADOs e insulina numa população, pois são menos onerosos, avalia a racionalidade da prescrição, o padrão de uso dos medicamentos, a adesão ao tratamento farmacológico, o perfil dos usuários portadores de DM, identificam as principais comorbidades associadas ao diabetes, além de outros fatores que apresentam fundamental importância na efetividade dos medicamentos, e que geralmente não podem ser observados em estudos pré-clínicos.

O presente estudo contribui fundamentalmente para a otimização do tratamento farmacológico do DM, pois fornece subsídios para a avaliação da utilização dos medicamentos ADOs e insulina nos pacientes atendidos pelo SUS no Distrito Sanitário Oeste do município de Ribeirão Preto-SP. 


\section{CONCLUSÕES}

"Leva-se muito tempo para não entender nada" (Edward Dahlberg). 
- A transição epidemiológica ocorrida no final do século XX indica que as doenças crônicas não-transmissíveis - dentre elas o Diabetes mellitus - serão consideradas as epidemias do século XXI. Aproximadamente $3,1 \%$ dos usuários do SUS do Distrito Sanitário Oeste de Ribeirão Preto-SP foram classificados como portadores de DM no período de mar/2006 a fev/2007. Estima-se que $14,4 \%$ da população acima de 60 anos pertencente ao Distrito Sanitário Oeste de Ribeirão Preto-SP são portadores dessa enfermidade. Essa prevalência atinge 18,0\% da população acima de 60 anos, caso seja utilizada a estimativa mundial de casos não diagnosticados.

- A idade média encontrada na população de estudo foi de 60,4 anos, sendo que, como esperado, os idosos constituem a maior parcela dessa população, correspondendo a 55,0\%. Além disso, observou-se uma proporção de 67 homens para 100 mulheres.

- A prevalência de DM1 foi de apenas $3,3 \%$ da população do estudo, abaixo dos valores descritos na literatura. Além disso, os indivíduos desse grupo apresentaram a menor média de idade do estudo.

- A monoterapia com antidiabéticos orais foi o esquema terapêutico mais prescrito durante o período de estudo, sendo a metformina o medicamento mais utilizado pelos usuários.

- Aproximadamente $96,5 \%$ dos usuários utilizaram as doses dos antidiabéticos orais e insulina dentro do intervalo terapêutico preconizado pelas diretrizes nacionais e internacionais.

- A adesão ao tratamento farmacológico, avaliada por meio da retirada de medicamentos juntos as farmácias das Unidades Básicas de Saúdes, foi superior a $70,0 \%$ para todos os Subgrupos Terapêuticos.

- Devido à complexidade do tratamento do DM, muitos pacientes apresentaram variação na dose e também no número de medicamentos durante o período de estudo. Aproximadamente $34,0 \%$ dos indivíduos apresentaram insucesso no tratamento farmacológico, enquanto que $9,8 \%$ obtiveram sucesso no tratamento. 
- O Grupo de usuários que utilizaram 2 medicamentos antidiabéticos orais para o tratamento do DM apresentou o maior índice no insucesso farmacológico, com um percentual de 35,5\% de usuários que necessitaram aumento de dose. Entretanto, o grupo de utilizou 2 medicamentos antidiabéticos orais mais insulina obteve o maior sucesso no tratamento farmacológico, apresentando retirada de pelo menos um medicamento em $47,5 \%$ dos usuários.

- Em relação às comorbidades, $73,5 \%$ são hipertensos e $25,9 \%$ dislipidêmicos, aumentando a probabilidade de ocorrência de complicações, mesmo quando tratados adequadamente, elevando o número de medicamentos e atendimento médico necessários, onerando o cuidado prestado ao paciente.

- Durante o período de estudo, os portadores de Diabetes mellitus utilizaram classes medicamentosas que podem interferir no controle glicêmico ou na efetividade dos antidiabéticos orais e/ou insulina, como por exemplo, a cimetidina, levotiroxina, fluconazol, propranolol, entre outros. Estes medicamentos devem ser administrados com cautela nos portadores de Diabetes mellitus.

- Todos os Grupos Terapêuticos apresentaram número médio de consulta igual a 7,0. Dessa forma, portadores de Diabetes mellitus que utilizavam monoterapia ou politerapia para o tratamento da enfermidade não apresentaram diferenciação no cuidado prestado pelo serviço público de saúdo do Distrito Sanitário Oeste de Ribeirão Preto-SP, mesmo apresentando perfis terapêuticos distintos e, portanto, condições clínicas diferentes.

- A necessidade de verificar a situação real do consumo de medicamentos e da prevalência das enfermidades faz com que os Estudos de Utilização de Medicamentos sejam considerados uma metodologia útil neste processo. Estes estudos podem descrever os padrões de uso de medicamentos, verificar variação no perfil terapêutico ao longo do tempo, estimar o número de indivíduos expostos a medicamentos, detectar doses excessivas, uso inadequado, doses insuficientes e utilização indiscriminada dos medicamentos, estimar as necessidades de medicamentos de uma sociedade, entre outras. 
- Os Estudos de Utilização de Medicamentos servem como informação importante para a construção dos guias (guidelines) práticos e contribuem para o direcionamento da prática médica e o uso racional dos medicamentos, garantindo uma terapêutica efetiva e segura. Além disso, são ferramentas adequadas e necessárias para a formulação de políticas de saúde, garantindo aos gestores de saúde o conhecimento do perfil epidemiológico das populações estudadas, identificando e prevenindo o uso irracional e reações adversas a medicamentos. 


\section{REFERÊNCIAS}

"O livro é um mestre que fala, mas que não responde" (Platão). 
ADA - AMERICAN DIABETES ASSOCIATION. Type 2 diabetes in children and adolescents. Diabetes Care, v. 23, no. 3, p. 381-389, 2000.

ADA - AMERICAN DIABETES ASSOCIATION. Hypertension Management in Adults with Diabetes. Diabetes Care, v.27, no. 1, p. S65-S67, 2004.

ADA - AMERICAN DIABETES ASSOCIATION. Nutrition Recommendations and Principles for People with Diabetes Mellitus. Diabetes Care, v.30, no. 1, p.S48-S65, 2007a.

ADA - AMERICAN DIABETES ASSOCIATION. Total prevalence of diabetes \& prédiabetes. Diabetes Statistics (2007b). Disponível em: <http://www.diabetes.org/ diabetes-statistics/prevalence.jsp>. Acesso em: 02 fev. 2009.

ADA - AMERICAN DIABETES ASSOCIATION. Diabetes facts and figures (2007c). Disponível em: <http://www.diabetes.org/diabetes-statistics.jsp>. Acesso em: 10 out. 2007.

ADA - AMERICAN DIABETES ASSOCIATION. Economic Costs of Diabetes in the U.S. in 2007. Diabetes Care, v. 31, no. 3, p. 1-20, 2008a.

ADA - AMERICAN DIABETES ASSOCIATION. Standards of Medical Care in Diabetes. Diabetes Care, v. 30, no. 1, p.S4-S41, $2008 \mathrm{~b}$.

ALBA, J.E.M; ESCOBAR, J.C.M; ESCOBAR, G.M. Patrones de prescripción de antidiabéticos em um grupo de pacientes colombianos. Revista Panamericana de Salud Pública, v. 22, no. 2, p. 124-131, 2007.

ALLHAT - THE ANTIHYPERTENSIVE AND LIPID-LOWERING TREATMENT TO PREVENT HEART ATTACK TRIAL. Major outcomes in high-risk hypertensive patients randomized to angiotensin-converting enzyme inhibitor or calcium channel blocker vs diuretic: The Antihypertensive and Lipid- Lowering Treatment to Prevent Heart Attack Trial (ALLHAT). Journal of the American Medical Association, v. 288, p. 2981-2997, 2002.

ALVARENGA, C. Hipertensão arterial na Diabetes Mellitus tipo 2 - evidência para a abordagem terapêutica. Revista Portuguesa de Clinica Geral, v. 21, p. 597-604, 2005. 
ARAÚJO, R.B; SANTOS, I.S; CAVALETI, M.A; DIAS DA COSTA, J.S; BERIA, J.U. Avaliação do cuidado prestado a pacientes diabéticos em nível primário. Revista de Saúde Pública, v. 33, no. 1, p.24-32, 1999.

ASCHNER, P. Diabetes trends in Latin America. Diabetes Metabolism Research and Reviews, v. 18, p. S27-S31, 2002.

BARCELÓ, A; ROBLES, S; WHITE, F; JADUE, L; VEJA, J. Uma interventión para mejorar el control de la diabetes em Chile. Revista Panamericana de Salud Pública, v. 10, no. 5, p. 328-333, 2001.

BARCELÓ A, AEDO C, SWAPNIL R, ROBLES S. The cost of diabetes in Latin America and the Caribbean. Bulletin of the World Health Organization, v. 81, no. 1, p. 19-27, 2003.

BJORNSSON, T.D Practical uses of individual pharmacokinetics parameters in drug development and clinical practice: examples and simulations. European Journal of Drug Metabolism and Pharmacokinetics. v. 22, no. 1, p. 1-14, 1997.

BOCCUZZI, S.J; WOGEN, J; FOX, J; SUNG, J.C.Y; SHAH, A.B; KIM, J. Utilization of Oral Hypoglycemic Agents in a Drug-Insured U.S. Population. Diabetes Care, v. 24, p. 1411-1415, 2001.

BRANDLE, M; ZHOU, H; SMITH, BRK; MARRIOTT, D; BURKE, R; TABAEI, B.P; BROWN, M.B; HERMAN, W.H. The Direct Medical Cost of Type 2 Diabetes. Diabetes Care, v. 26, no. 8, p. 2300-2304, 2003.

BRASIL. Constituição (1988). Constituição da República Federativa do Brasil. Brasília, DF: Senado, 5 out. 1988. Seção 1, p.1.

BRASIL. Lei n.8080, de 19 de setembro de 1990. Dispõe sobre as condições para a promoção, proteção e recuperação da saúde, a organização e o funcionamento dos serviços correspondentes e dá outras providências. Diário Oficial da União, Brasília, DF, 19 set. 1990a.

BRASIL. Lei n.8142, de 28 de dezembro de 1990. Dispõe sobre a participação da comunidade na gestão do Sistema Único de Saúde - SUS e sobre as transferências intergovernamentais de recursos financeiros na área da saúde e dá outras providências. Diário Oficial da União, Brasília, DF, 28 dez. 1990b. 
BRASIL. Portaria n.371/GM, de 04 de março de 2002. Instituir o Programa Nacional de Assistência Farmacêutica para Hipertensão Arterial e Diabetes Mellitus, parte integrante do Plano Nacional de Reorganização da Atenção a Hipertensão Arterial e Diabetes Mellitus. Diário Oficial da União, Brasília, DF, 04 mar. 2002.

BRASIL. Portaria n. 399/GM, de 22 de fevereiro de 2006. Divulga o Pacto pela Saúde 2006 - Consolidação do SUS e aprova as Diretrizes Operacionais do Referido Pacto. Diário Oficial da União, Brasília, DF, 22 fev. 2006a.

BRASIL. Conselho Nacional de Secretários de Saúde. SUS: avanços e desafios. $1^{\circ}$ ed. Brasília, 2006b. 164p.

BROWN, J.B; NICHOLS, G.A; GLAUBER, H.S; BAKST, A. Ten-Year Follow-up of Antidiabetic Drug Use, Nonadherence, and Mortality in a Defined Population with Type 2 Diabetes Mellitus. Clinical Therapeutics, v. 21, no. 6, p. 1045-1055, 1999.

BURGERS, J.S; BAILEY, J.V; KLAZINGA, N.S; VAN DER BIJ, A.K; GROL, R; FEDER, G. For the AGREE Collaboration. Inside guidelines: Comparative analysis of recommendations and evidence in diabetes guidelines from 13 countries. Diabetes Care, v. 25, no. 11, p. 1933-1939, 2002.

CAPORALE, J.E; CALVO, H; GAGLIARDINO, J.J. Costos de atención médica de personas con diabetes anteriores y posteriores a su hospitalización en Argentina. Revista Panamericana de Salud Pública, v. 20, no. 6, p. 361-368, 2006.

CARVALHO, A.I. Conselhos de Saúde no Brasil: participação cidadã e controle social. Rio de Janeiro: Fase/lbam, 1995. 136p.

CASTRO, L.L.C. Farmacoepidemiologia: uma nova disciplina. In: Fundamentos de Farmacoepidemiologia. Campo Grande: GRUPURAM, $\overline{2000, p}$. 6-18.

CHENG, S. F; HSU, H.H; LEE, H.S; LIN, C.S; CHOU, Y.C; TIEN, J. H. Rational pharmacotherapy in the diabetic hypertension: analysis-prescribing patterns in a general hospital in Taiwan. Journal of Clinical Pharmacy and Therapeutics, v. 29, p. 547-558, 2004.

CHIANG, C. W; CHIU, H. F; CHEN, C. Y; WU, H. L; YANG, C. Y. Trends in the use of oral antidiabetic drugs by outpatients in Taiwan: 1997-2003. Journal of Clinical Pharmacy and Therapeutics, v. 31, p. 73-82, 2006. 
CODE-2 - COST OF DIABETES IN EUROPE TYPE 2. Revealing the costs of type 2 diabetes in Europe. EASD Satellite Symposium Report, Brussels, 27 september, 1999.

CONSENSO LATINO-AMERICANO DE OBESIDADE. Arquivos Brasileiros de Endocrinologia e Metabologia, v. 43, no. 1, p. 21-67, 1998.

CROZARA, M.A. Estudo do consumo de medicamentos em hospital particular. São Paulo, 2001. 133 p. [Dissertação de Mestrado - Faculdade de Ciências Farmacêuticas - Universidade de São Paulo].

DAWSON, D; CONLON, C. Case study: Metformin associated lactic acidosis: Could orlistat be relevant? Diabetes Care, v. 26, no. 8, p. 2471-2472, 2003.

DCCT- DIABETES CONTROL AND COMPLICATIONS TRIAL RESEARCH GROUP. The effect of intensive treatment of diabetes on the development and progression of long-term complications in insulin-dependent diabetes mellitus. Journal of the American Medical Association, v. 276, p. 1409-1415, 1996.

DEFRONZO, R.A. Pharmacology therapy for type 2 diabetes mellitus. Annals of Internal Medicine, v. 131, p. 281-303, 1999.

DERI - DIABETES EPIDEMIOLOGY RESEARCH INTERNATIONAL STUDY. International analysis of insulindependent diabetes mellitus mortality: a preventable mortality perspective. American Journal of Epidemiology, v. 142, p. 612-618. 1995.

DORÓ, P; BENKÖ, R; KOSIK, E; MATUZ, M; TÓTH, K; SOÓS, G. Utilization of oral antihyperglycemic drugs over a 7-year period (1998-2004) in a Hungarian population and adherence to drug therapy. European Journal of Clinical Pharmacology, v. 61, p. 893-897, 2005.

DPP - DIABETES PREVENTION PROGRAM GROUP. Reduction in the incidence of type 2 diabetes with lifestyle intervention or metformin. New England Journal of Medicine, v. 346, p. 393-403, 2002.

DUARTE-RAMOS, F; CABRITA, J. Using a pharmacoepidemiological approach to estimate diabetes type 2 prevalence in Portugal. Pharmacoepidemiology and Drug Safety, v. 15, p.269-274, 2006. 
ESCOREL, S. Reviravolta na Saúde: origem e articulação do movimento sanitário. Rio de Janeiro: Fiocruz, 1998. 206p.

ESPELAND, M.A; HOGAN, P.E; FINEBERG, S.E; HOWARD, G. SCHROTT, H; WACLAWIW, M.A; BUSH, T.L. Effect of postmenopausal hormone therapy on glucose and insulin concentrations. Diabetes Care, v. 21, no. 10, p. 1589-1595, 1998.

FRANCO, L.J; ROCHA, J.S.Y. O aumento das hospitalizações por diabetes na região de Ribeirão Preto, SP, no período de 1988-97. Diabetes Clínica, v. 6, p.108, 2002.

GILMER, T.P; O'CONNOR, P.J; MANNING, W.G; RUSH, W.A. The cost to health plans of poor glycemic control. Diabetes Care, v. 20, no. 12, p. 1847- 1853, 1997.

GOMES, M.B; GIANNELLA, N.D; MENDONÇA, E; TAMBASCIA, M.A; FONSECA, R.M; RAEA, R.R; MACEDO, G; MODESTO-FILHO, J; SCHMID, H; BITTENCOURT, A.V; CAVALCANTI, S; RASSI, N; FARIA, M; PEDROSA, H; DIB, S.A.Prevalência de Sobrepeso e Obesidade em Pacientes Com Diabetes Mellitus do Tipo 2 no Brasil: Estudo Multicêntrico Nacional. Arquivos Brasileiros de Endocrinologia \& Metabologia, v. 50, no. 1, p. 136-144, 2006.

GRANT RW, DEVITA NG, SINGER DE, MEIGS JB. Polypharmacy and medication adherence in patients with type 2 diabetes. Diabetes Care, v. 26, no. 5, p. 14081412, 2003.

GRESS, T.W; NIETO, F.J; SHAHAR, E. WOFFORD, M.R; BRANCATI, F.L. Hypertension and antihypertensive therapy as risk factors for type 2 diabetes mellitus. New England Journal of Medicine, v. 342, p. 905-912, 2000.

GRIMALDIA, A; GRANGÉ, V; ALLANNICC, H; PASSAD, P; RODIERE, M; CORNETF, P; DUPRATB, I; DUC-DODONG, P; LEMAIREH, A; LIARDI, F; ESCHWEGE, E. Epidemiological analysis of patients with Type 2 diabetes in France. Journal of Diabetes and Its Complications, v. 14, p. 242-249, 2000.

GRUNDY, S.M; BREWER, B; CLEEMAN, J.I; SMITH, S.C; LENFANT, C. Report of the National Heart, Lung, and Blood Institute/American Heart Association Conference on Scientific Issues Related to Definition. Circulation, v. 109, p. 433-438, 2004. 
GU, K; COWIE, C.C; HARRIS, M.I. Mortality in adults with and without diabetes in a national cohort of the US population, 1971-1993. Diabetes Care, v. 21, no. 7, p. 1138-1145, 1998.

GUIDONI, C.M; OLIVERA, C.M.X; FREITAS, O; PEREIRA, L.R.L. Diabetes Mellitus e Sistema Único de Saúde: Análise do Modelo Atual. Brazilian Journal of Pharmaceutical Sciences, v. 45, n. 1, 2009. In Press.

GUS, M; FUCHS, F.D. Análise crítica das evidências sobre o tratamento da hipertensão arterial no paciente com diabetes melito tipo 2. Revista Brasileira de Hipertensão, v. 13, no. 3, p. 193-197, 2006.

HAAJER-RUSKAMP, F; HEMMINKI, E. The social aspects of drug use. In: Drug utilization studies: methods and uses. Copenhagen, OMS, Regional Office for Europe, p. 97-123, 1993.

HALLAS, J. Conducting pharmacoepidemiologic research in Denmark. Pharmacoepidemiology and Drug Safety, v. 10, p. 619-623, 2001.

HARRIS, M.I. Health care and health status and outcomes for patients with type 2 diabetes. Diabetes Care, v. 23, no. 6, p. 754-758, 2000.

HARTZEMA, G; PORTA, M.S; HUGH, H.T. Pharmacoepidemiology: An Introduction. $3^{\circ}$ ed. Cincinnati OH: Harvey Whitney Books Company, 1998. 670 p.

KALANTARIDOU, S.N; DAVIS, S.R; CALIS, K.A. Hormone Therapy in Women. In: DIPIRO, J.T; TALBERT, R.L; YEE, G.C; MATZKE, G.R; WELLS, B.G; POSEY, L.M. Pharmacotherapy: A Pathophysiologic Approach. 7ed. Estados Unidos: McGrawHill, p. p. 1351-1368, 2008.

KANAYA, A.M; HERRINGTON, D; VITTINGHOFF, E; LIN, F; GRADY, D; BITTNER, V; CAULEY, J.A; BARRETT-CONNOR, E. Glycemic effects of postmenopausal hormone therapy: The Heart and Estrogen/Progestin Replacement Study. A randomized, double-blind, placebo-controlled trial. Annals of Internal Medicine, v. 138, p. 01-09, 2003.

HENRIKSSON, F; AGARDH, C; BERNE, C. Direct medical costs for patients with type 2 diabetes in Sweden. Journal of Internal Medicine, v. 248, p.387-96, 2000. 
HENRY, D.A; MCGETTIGAN, P; TONKINN, A; HENNESSY, S. Basic Principles of Clinical Pharmacology Relevant to Pharmacoepidemiology Studies. In STROM, B.L. Pharmacoepidemiology. 3ํe․ John Wiley \& Sons: Chichester, p. 37-57, 2000.

HOGAN, P; DALL, T; NIKOLOV, P. Economic costs of diabetes in the US in 2002. Diabetes Care, v. 26, no. 3, p. 917-932, 2003.

HUMPHREY, L.L; PALUMBO, P.J; BUTTERS, M.A; HALLETT JR, J.W; CHU, C.P; O'FALLON, W.M; BALLARD, D.J. The contribution of non-insulin-dependent diabetes to lower extremity amputation in the community. Archives of Internal Medicine, v. 154, p.885-892, 1994.

HUNDAL, R.S; INZUCCHI, S.E. Metformin: New Understandings, New Uses. Drugs, v. 63 , no. 18 , p. 1879-1894, 2003.

IBGE - INSTITUTO BRASILEIRO DE GEOGRAFIA E ESTATÍSTICA. Contagem da população, 2007. 311p.

IBGE - INSTITUTO BRASILEIRO DE GEOGRAFIA E ESTATÍSTICA. Síntese de indicadores sociais: uma análise das condições de vida da população brasileira. Estudos e pesquisa: Informação Demográfica e Socioeconômica, no. 23, 2008. 280p.

IDF - INTERNATIONAL DIABETES FEDERATION. Diabetes Atlas. 3ํe ed. Brussel, 2006. Backgrounder. Disponível em: <http://www.eatlas.idf.org/webdata/docs/ backgroundopeningpc.pdf>. Acesso em: 07 jun. 2007.

JAWAD, M.K; GREGORY, Y.H.L. The management of hypertension in type II diabetes mellitus. Revista Brasileira de Hipertensão, v. 13, no. 1, p. 8-13, 2006.

KING, H; AUBERT, R.E; HERMAN, W.H. Global burden of diabetes, 1995-2025. Diabetes Care, v. 21, no. 9, p.1414-1431, 1998.

KLAASSEN, C.D. Princípios de toxicologia e Tratamento do envenenamento. In: GOODMAN, L.S; GILMAN, A. As bases farmacológicas da terapêutica. $10^{\circ} \mathrm{ed}$. Rio de Janeiro: Guanabara Koogan, p. 51-61, 2006.

KONDRO, W. Drug spending hits $\$ 24.8$ billion. Canadian Medical Association Journal, v. 175, no. 1, p. 22, 2006. 
LAPORTE, J.R; TOGNONI, G; ROSENFELD, S. Epidemiologia do medicamento: princípios gerais. São Paulo: Hucitec-Abrasco, 1989. 293p.

LAZAROU, J.; POMERANZ, B.H; COREY, P.N. Incidence of adverse drug reactions in hospitalized patients: a meta-analysis of prospective studies. Journal of the American Medical Association, v. 279, p. 1200-1205, 1998.

LEE, D; BERGMAN, U. Studies of Drug Utilization. In STROM, B.L. Pharmacoepidemiology. 3o ed. John Wiley \& Sons: Chichester, p. 401-417, 2000.

LENZ, W. Thalidomide: facts and inferences. In: LAPORTE, J.R; TOGNONI, G; ROSENFELD, S. Epidemiologia do medicamento: princípios gerais. São Paulo: Hucitec-Abrasco, p. 43-55, 1989.

MACDONALD, S. Increased drug spending is creating funding crisis, report says. British Medical Journal, v. 326, p. 677, 2003.

MALERBI, D.A; FRANCO, L.J. Multicenter study of the prevalence of diabetes mellitus and impaired glucose tolerance in the urban Brazilian population aged 30-69 Years. Diabetes Care, v. 15, p. 1509-1516, 1992.

MALTA, D.C; CEZÁRIO, A.C; MOURA, L; MORAIS-NETO, O.L; SILVA-JUNIOR, J.B. A construção da vigilância e prevenção das doenças crônicas não transmissíveis no contexto do Sistema Único de Saúde. Caderno de Saúde Pública, v. 15, no. 1, p.47-65, 2006.

MARIN, N; LUIZA, V.L; OSORIO-DE-CASTRO, C.G.S; MACHADO-DOS-SANTOS, $\mathrm{S}$. Assistência farmacêutica para gerentes municipais de saúde. Rio de Janeiro: OPAS/OMS; 2003. 373p.

MELANDER, A; FOLINO-GALLO, P; WALLEY, T; SCHWABE, U; GROOP, P.H; KLAUKKA, T; VALLANO, A; LAPORTE, J.R; GALLEGO, M.R; SCHIAPPA, M; RØDER, M; KAMPMANN, J.P; SWAEF, A; ÅBERG, M; MÅNSSON, N.O; LINDBLAD, $U$. Utilisation of antihyperglycaemic drugs in ten European countries: different developments and different levels. Diabetologia, v. 49, p. 2024-2029, 2006.

MELLO-JORGE, M.H.P; GOTLIEB, S.L.D; LAURENTI, R. A saúde no Brasil: análise do período 1996 a 1999. Brasília: OPAS, 2001. 238p. 
MENDOZA RUIZ, A.; OSÓRIO-DE-CASTRO, C.G.S. Medicamentos: falando de qualidade. Organizadores: Gabriela Costa Chaves, Renata Reis, Veriano Terto Jr., Cristina Pimenta. 1ed. Rio de Janeiro: ABIA, 2008. 52p.

MICROMEDEX® Healthcare Series. Glyburide. (2009a). Disponível em: <http://www.thomsonhc.com/hcs/librarian/ND_T/HCS/ND_PR/Main/CS/F05A60/DUP LICATIONSHIELDSYNC/22AF90/ND_PG/PRIH/ND_B/HCS/SBK/2/ND_P/Main/PFP Ul/Jw16huU2Palhzy/PFActionld/hcs.common.RetrieveDocumentCommon/Docld/059 2/ContentSetld/31/SearchTerm/glyburide\%20/SearchOption/BeginWith>. Acesso em: 30 jan. 2009.

MICROMEDEX® Healthcare Series. Gliclazide. (2009b). Disponível em: $<$ http://www.thomsonhc.com/hcs/librarian/ND_T/HCS/ND_PR/Main/CS/F05A60/DUP LICATIONSHIELDSYNC/22AF90/ND_PG/PRIH/ND_B/HCS/SBK/5/ND_P/Main/PFP Ul/Jw16huU2PaK3o6/PFActionld/hcs.common.RetrieveDocumentCommon/Docld/15 17/ContentSetld/31/SearchTerm/gliclazide\%20/SearchOption/BeginWith>. Acesso em: 30 jan. 2009.

MICROMEDEX® Healthcare Series. Metformin. (2009c). Disponível em: <http://www.thomsonhc.com/hcs/librarian/ND_T/HCS/ND_PR/Main/CS/F05A60/DUP LICATIONSHIELDSYNC/22AF90/ND_PG/PRIH/ND_B/HCS/SBK/8/ND_P/Main/PFP Ul/Jw16huU2PaKkTd/PFActionld/hcs.common.RetrieveDocumentCommon/Docld/04 46/ContentSetld/31/SearchTerm/metformin\%20/SearchOption/BeginWith>. Acesso em: 30 jan. 2009.

MICROMEDEX® Healthcare Series. Insulin. (2009d). Disponível em: $<$ http://www.thomsonhc.com/hcs/librarian/ND_T/HCS/ND_PR/Main/CS/F05A60/DUP LICATIONSHIELDSYNC/22AF90/ND_PG/PRIH/ND_B/HCS/SBK/11/ND_P/Main/PFP $\mathrm{UI} / \mathrm{Jw}$ 16huU2PaKHuM/PFActionld/hcs.common.RetrieveDocumentCommon/Docld/0 002/ContentSetld/31/SearchTerm/insulin\%20/SearchOption/BeginWith>. Acesso em: 30 jan. 2009.

MICROMEDEX® Healthcare Series (2009e). Disponível em: <https://www. thomsonhc.com/hcs/librarian/>. Acesso em: 30 jan. 2009.

MILES, J.M; LEITER, L; HOLLANDER, P; WADDEN, T; ANDERSON, J.W; DOYLE, M; FOREYT, J; ARONNE, L; KLEIN, S. Effect of orlistat in overweight and obese patients with type 2 diabetes treated with metformin. Diabetes Care, v. 25, no. 7, p. 1123-1128, 2002. 
MS - MINISTÉRIO DA SAÚDE (Brasil). Prevenção e controle das doenças não transmissíveis no Brasil. Brasília, DF, editora MS, 2002a. (Textos Básicos de Saúde. Série B).

MS - MINISTÉRIO DA SAÚDE (Brasil). Manual de hipertensão arterial e diabetes mellitus. Brasília, DF, editora MS; 2002b. 102p. (Projetos, Programas e Relatórios. Série C, n. 59).

MS - MINISTÉRIO DA SAÚDE (Brasil). Secretaria de Vigilância em Saúde. Departamento de Análise de Situação de Saúde. A vigilância, o controle e a prevenção das doenças crônicas não transmissíveis. DCNT no contexto do Sistema Único de saúde brasileiro. Brasília: OPAS, 2005. 80p. (Série: Situação e Desafios Atuais).

MS - MINISTÉRIO DA SAÚDE. Secretaria de Atenção à Saúde. Departamento de Atenção Básica. Diabetes Mellitus. Brasília, DF, editora MS, 2006a. 64p.

MS - Ministério da Saúde (Brasil). Secretaria de Gestão Estratégica e Participativa. A construção do SUS: histórias da Reforma Sanitária e do Processo Participativo. Brasília, DF, editora MS, 2006b. 300 p. (Série I. História da Saúde no Brasil).

MS - MINISTÉRIO DA SAÚDE (Brasil) (2007a). Hiperdia: Sistema de Cadastramento e Acompanhamento de hipertensos e diabéticos. Disponível em: <http://hiperdia. datasus.gov.br/.>. Acesso em: 10 jun. 2007.

MS - MINISTÉRIO DA SAÚDE (Brasil). Área de Economia da Saúde e Desenvolvimento. Núcleo Nacional de Economia da Saúde. Evolução dos Gastos do Ministério da Saúde com Medicamentos. Brasília, DF, editora MS, 2007b. 34p.

MODESTO-FILHO, J; VASCONCELOS, T.N.C; FERNANDES, A.C.C; FRANÇA, J. Diabetes e hospitalização: Dados do Hospital Unimed - JP. Diabetes Clínica, v. 11, no. 1, p. 68, 2007.

MOLENA-FERNADES, C.A; NARDO, N.J; TASCA, R.S; PELLOSO, S.M; CUMAN, R.K.N. A importância da associação de dieta e de atividade física na prevenção e controle do Diabetes mellitus tipo 2. Acta Scientiarum Health Science, v. 27, no. 2, p.195-205, 2005. 
MONNIER, L; BENICHOU, M; CHARRA-EBRARD, S; BOEGNER, C; COLETTE, C. An overview of the rationale for pharmacological strategies in type 2 diabetes: from the evidence to new perspectives. Diabetes Metabolism, v. 31, p. 101-109, 2005.

MORRISH, N.J; WANG, S.L; STEVENS, L.K; FULLER, J.H; KEEN, H. Mortality and causes of death in the OMS Multinational study of vascular disease in diabetes. Diabetologia, v. 44, no. 2, p. S14-S21, 2001.

NIDDK - National Institute of Diabetes and Digestive and Kidney Diseases. The National Diabetes Information Clearinghouse. National Diabetes Statistics, 2007. $24 p$.

OMS - ORGANIZAÇÃO MUNDIAL DE SAÚDE. La seleción de medicamentos essenciales. Genebra, OMS, 1977. 6p. (Série Informe Técnicos, n.615).

OMS - ORGANIZAÇÃO MUNDIAL DE SAÚDE. Obesity: preventing and managing the global epidemic: report of a OMS consultation on obesity, 3-5 June 1997. Geneva, Switzerland: OMS, 1998.

OMS - ORGANIZAÇÃO MUNDIAL DE SAÚDE. Definition, diagnosis and classification of diabetes mellitus and its complications: report of OMS consultation, 1999. Disponível em: <http://www.OMS.int/diabetes/publications/ Definition\%20and\%20diagnosis\%20of\%20diabetes_new.pdf>. Acesso em: 15 mai. 2007.

OMS - ORGANIZAÇÃO MUNDIAL DE SAÚDE. National Pharmacovigilance Systems country Profiles and Overview. Uppsala: The Uppsala Monitoring Centre (1997), 2002.

OMS - ORGANIZAÇÃO MUNDIAL DE SAÚDE. Introduction to drug utilization research. WHO International Working Centre for Drug Statistics Methodology, WHO Collaborating Centre for Drug Utilization Research and Clinical Pharmacological Services, 2003a. 48p.

OMS - ORGANIZAÇÃO MUNDIAL DE SAÚDE. Diet, nutrition and the prevention of chronic diseases: Report of a Joint OMS Expert Consultation. Geneva: OMS, 2003b. (Technical Report Series n. 916).

OMS - ORGANIZAÇÃO MUNDIAL DE SAÚDE. How to investigate the use of medicines by consumers. Geneva, OMS, 2004. 98p. 
OMS - ORGANIZAÇÃO MUNDIAL DE SAÚDE. Preventing chronic diseases: a vital investment. Geneva: OMS, Public Health Agency of Canada, 2005. 182p.

OPAS - ORGANIZAÇÃO PAN-AMERICANA DA SAÚDE; ALAD - ASOCIACIÓN LATINOAMERICANA DE DIABETES. The Costs of Diabetes in the Americas. Synposium on Diabetes Economics; 27 September 2004; São Paulo, Brasil, p. 0103, 2004.

OPAS - ORGANIZAÇÃO PAN-AMERICANA DA SAÚDE. Prevenção de doenças crônicas: um investimento vital. Vigilância, o Controle e a Prevenção das Doenças Crônicas Não Transmissíveis (DCNT) no Contexto do Sistema Único de Saúde Brasileiro - Situação e Desafios Atuais. Brasília: OMS, 2005. 80p.

OPAS - ORGANIZAÇÃO PAN-AMERICANA DE SAÚDE. Saúde nas Américas. Washington, DC: OPAS; 2007. 453p. (OPAS, Publicação Científica e Técnica no. $622)$.

OPAS - ORGANIZAÇÃO PAN-AMERICANA DE SAÚDE. Guías ALAD de diagnóstico, control y tratamiento de Diabetes Mellitus tipo 2. Washington, DC, 2008. 82p.

OSÓRIO-DE-CASTRO, C.G.S (coord.). Estudo de utilização de medicamentos: noções básicas. Rio de Janeiro: Editora Fiocruz, 2000. 92p.

PAES, A.H.P; BAKKER, A; SOE-AGNIE, C.J. Impact of Dosage Frequency on Patient Compliance. Diabetes Care, v. 20, no. 10, p. 1512-1517, 1997.

PASSA, P. Diabetes trends in Europe. Diabetes/metabolism research and reviews, v. 18, p. S3-S8, 2002.

PERNEGER, T.V; BRANCATI, F.L; WHELTON, P.K; KLAG, M.J. End-stage renal disease attributable to diabetes mellitus. Annals of Internal Medicine, v. 121, p. 912-918, 1994.

PMRP - PREFEITURA MUNICIPAL DE RIBEIRÃO PRETO. Secretaria da Fazenda (2007). Disponível em: <http://www.ribeiraopreto.sp.gov.br/PRINCIPALN.php? pagina=/FAZENDA/i30PRINCIPAL.HTM>. Acesso em: 02 mar. 2007. 
P.R. Vade-mécum (2009). Disponível em: <http://www.prvademecum. com/prvademecumbra/bradefault.asp >. Acesso em: 30 jan. 2009.

PSATY, B.M; FURBERG, C.D. COX-2 inhibitors: Lessons in drug safety. New England Journal of Medicine, v. 352, p. 1133-1135, 2005.

RENDELL, M. The Role of Sulphonylureas in the Management of Type 2 Diabetes Mellitus. Drugs, v. 64, no. 12, p. 1339-1358, 2004.

RICHARDSON, S.J; SENIKAS, J.F; NELSON, J.F. Follicular depletion during the menopausal transition: Evidence for accelerated loss and ultimate exhaustion. Journal of Clinical Endocrinology \& Metabolism, v. 65, p. 1231-1237, 1987.

RIDDLE, M. Combining Sulfonylureas and Other Oral Agents. The American Journal of Medicine, v. 108, no. 6A, p. 15S-22S, 2000.

RODRIGUEZ NETO, E. Reunião da Abrasco em Cachoeira, Bahia. Saúde em Debate, Londrina, p.33-38, 1988.

ROTHMAN KJ, GREENLAND S. Types of epidemiologic study. In: Modern Epidemiology. $2^{\circ}$ ed. Philadelphia: Lippincott williams \& Wilkins, p. $\overline{67-78,1998 .}$

ROZENFELD, S; RANGEL, I. T. M. A farmacovigilância. Caderno de Saúde Pública, v. 4, n. 3, p. 336-341, 1988.

SARTORELLI, D.S; FRANCO, L.J. Tendências do diabetes mellitus no Brasil: o papel da transição nutricional. Caderno de Saúde Pública, v. 19, no. 1, p. S29-S36, 2003.

SARTORELLI, D.S; FRANCO, L.J; CARDOSO, M.A. Intervenção nutricional e prevenção primária do diabetes mellitus tipo 2: uma revisão sistemática. Caderno de Saúde Publica, v. 22, no. 1, p.7-18, 2006.

SBD - SOCIEDADE BRASILEIRA DE DIABETES. Consenso brasileiro sobre diabetes 2002: diagnóstico e classificação do diabetes melito e tratamento do diabetes melito do tipo 2. Rio de Janeiro: Editora Diagraphic, 2003. 72p. 
SBD - SOCIEDADE BRASILEIRA DE DIABETES. Tratamento e acompanhamento do Diabetes mellitus. Diretrizes da Sociedade Brasileira de Diabetes, 2006. 154p

SCHRAMM, J.M.A; OLIVEIRA, A.F; LEITE, I.C; VALENTE, J.G; GADELHA, A.M.J; PORTELA, M.C; CAMPOS, M.R. Transição epidemiológica e o estudo da carga de doença no Brasil. Ciência e Saúde Coletiva, v. 9, no. 4, p.897-908, 2004.

SHALANSKY SJ, LEVY AR. Effect of number of medications on cardiovascular therapy adherence. Annals of Pharmacotherapy, v. 36, no. 10, p. 1532-1539, 2002.

SMS-RP - SECRETARIA MUNICIPAL DE SAÚDE DE RIBEIRÃO PRETO (2008). Disponível em: <http://www.ribeiraopreto.sp.gov.br/ssaude/i16principal.asp?pagina= /saude/Conselho/l16indice.htm>. Acesso em: 09 jun. 2008.

SOBRAVIME - Sociedade Brasileira de Vigilância de Medicamentos - Estatuto da sociedade brasileira de vigilância de medicamentos. Boletim número 1, dez-jan, 1991.

SPITZ, A; HEYMSFELD, S; BRANK, R.C. Drug therapy for obesity: clinical considerations. Endocrine Practice, v. 1, p. 274-279, 1995.

STROM, B.L. What is pharmacoepidemiology? In: Pharmacoepidemiology. 3 o ed. John Wiley \& Sons: Chichester, p. 3-15, 2000.

TORQUATO, M.T; MONTENEGRO JÚNIOR, R.M; VIANA, L.A; DE SOUZA, R.A; LANNA, C.M; LUCAS, J.C; BIDURIN, C; FOSS, M.C. Prevalence of diabetes mellitus and impaired glucose tolerance in the urban population aged 30-69 years in Ribeirão Preto (São Paulo), Brazil. Medical Journal, v. 121, no. 6, p. 224-230, 2003.

TOSCANO, C.M. As campanhas nacionais para detecção das doenças crônicas não-transmissíveis: diabetes e hipertensão arterial. Ciência e Saúde Coletiva, v. 9, no. 4, p. 885-895, 2004.

TRIPLITT, C.L; REASNER, C.A; ISLEY, W.L. Diabetes mellitus. In: DIPIRO, J.T; TALBERT, R.L; YEE, G.C; MATZKE, G.R; WELLS, B.G; POSEY, L.M. Pharmacotherapy: A Pathophysiologic Approach. 7ed. Estados Unidos: McGrawHill, p. 1205-1242, 2008. 
TURNER, R.C. The UK Prospective Diabetes Study. Diabetes Care, v. 21, no. 3, p. C35-C38, 1998.

TURNER, R,C; CULL, C.A; FRIGHI, V; HOLMAN, R.R. Glycemic control with diet, sulfonylurea, metformin, or insulin in patients with type 2 diabetes mellitus: progressive requirement for multiple therapies. Journal of the American Medical Association, v. 281, p. 2005-2012, 1999.

UKPDS - UNITED KINGDOM PROSPECTIVE DIABETES STUDY. Overview of 6 years' therapy of type 2 diabetes: a progressive disease. Diabetes, v. 44, no. 11 , p. 1249-1258, 1995.

UKPDS - UNITED KINGDOM PROSPECTIVE DIABETES STUDY GROUP. Effect of intensive blood-glucose control with metformin on complications in overweight patients with type 2 diabetes (UKPDS 34). Lancet, v. 352, p. 854-865, 1998a.

UKPDS - UNITED KINGDOM PROSPECTIVE DIABETES STUDY GROUP. Tight blood pressure control and the risk of macrovascular and microvascular complications in type diabetes (UKPDS 38). British Medical Journal, v. 317, p. $703-713,1998 b$.

UKPDS - UNITED KINGDOM PROSPECTIVE DIABETES STUDY GROUP. Intensive blood glucose control with sulphonylureas or insulin compared with conventional treatment and risk of complications in patients with type 2 diabetes (UKPDS 33). Lancet, v. 352, p. 837 -853, 1998c.

VAUZELLE-KERVROËDAN, F; JAVOY, F; FORHAN, A; FENDER, P; ESCHWĖGE, $E$. Pharmacoépidémiologie du diabète: évaluation du bon usage des antidiabétiques oraux. Diabetes \& Metabolism, v. 26, p. 63-68, 2000.

WANDELL, P. E; GAFVELS, C. Drug prescription in men and women with type-2 diabetes in Stockholm in 1995 and 2001:change over time. Europe Journal of Clinical Pharmacology, v. 58, p. 547-553, 2002.

WERTHEIMERA, A.I; ANDRES, K.B. An overview of pharmacoepidemiology. Pharmacy World Science, v. 17, no. 3, p. 61-66, 1995.

WIWANITKIT, V. Diabetes type 3: A brief review. Diabetes \& Metabolic Syndrome: Clinical Research \& Reviews, v. 2, p. 223-226, 2008. 